Zbl Arbeitsmed 2014 $\cdot 64: 10-25$ DOI 10.1007/s40664-013-0001-5

Online publiziert: 6. Februar 2014

(c) Springer-Verlag Berlin Heidelberg 2014

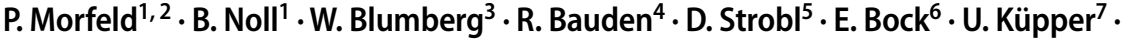
E. Gross ${ }^{8} \cdot$ S. Schauhoff ${ }^{8}$

${ }^{1}$ Institut für Epidemiologie und Risikobewertung in der Arbeitswelt, Evonik Industries AG, Essen

${ }^{2}$ Institut und Poliklinik für Arbeitsmedizin, Umweltmedizin und Präventionsforschung, Universität zu Köln

${ }^{3}$ Business Unit Advanced Intermediates, Evonik Degussa Antwerpen N.V., Antwerpen

${ }^{4}$ Medische Dienst, Evonik Degussa Antwerpen N.V., Antwerpen

${ }^{5}$ Business Unit Advanced Intermediates, Evonik Industries AG, Münchsmünster

${ }^{6}$ Werksärztlicher Dienst, Thyssen Krupp, Münchsmünster

${ }^{7}$ Werksärztlicher Dienst, Evonik Industries AG, Wesseling

${ }^{8}$ Business Unit Advanced Intermediates, Evonik Industries AG, Wolfgang

\title{
Cyanurchloridbelastung in Produktionsbetrieben
}

\section{Teil 1: Kollektivbeschreibung, Expositionsdatenaufbereitung sowie Erhebung medizinischer Response-Größen und wichtiger Kovariablen}

Cyanurchlorid $\left(\mathrm{C}_{3} \mathrm{Cl}_{3} \mathrm{~N}_{3}\right)$ ist ein weißes kristallines Pulver. Es wird für die Herstellung von Herbiziden benötigt, die in der Landwirtschaft eingesetzt werden. Weitere Verwendung findet es in der Produktion von Textilfarbstoffen und optischen Aufhellern oder der Herstellung von UVStabilisatoren für die Plastikindustrie sowie insbesondere für Automobilanwendungen in der Gummiindustrie.

Zurzeit gibt es keinen Arbeitsplatzgrenzwert für die Chemikalie Cyanurchlorid, sodass die Auswirkung einer Cyanurchloridbelastung auf die Gesundheit des Menschen nur eingeschränkt beurteilt werden kann [11]. Gegenstand dieser epidemiologischen Studie ist es zu prüfen, ob in einer Studiengruppe von Mitarbeitern in Cyanurchloridbetrieben der Evonik Industries AG über den Studienzeitraum 1958-2007 nachteilige Einflüsse der Cyanurchloridbelastung insbesondere auf ausgewählte Lungenfunktionsparameter festzustellen sind und hieraus einen Vorschlag für einen Arbeitsplatzgrenzwert (AGW) abzuleiten.
Cyanurchlorid ist in den Fokus der maximale Arbeitsplatzkonzentration(MAK)Kommission (Senatskommission zur Prüfung gesundheitsschädlicher Arbeitsstoffe der Deutschen Forschungsgemeinschaft) geraten, die regelmäßig auch Stoffe bewertet, für die noch keine MAK festgelegt wurden [21]. Darüber hinaus ist auch eine Einordnung von Cyanurchlorid im Rahmen der REACH-/CLP (Registration, Evaluation, Authorisation and Restriction of Chemicals/Classification, Labelling, Packaging)-Verordnungen in nächster Zukunft zu erwarten [13, 14].

Cyanurchlorid ist ein heterozyklisches Zwischenprodukt und wird im großtechnischen Maßstab hergestellt. Cyanurchlorid eignet sich zur Herstellung vieler Produkte. Durch die Möglichkeit der selektiven Substitution der 3 Chloratome sowie durch die große Stabilität des Triazinrings können mit Cyanurchlorid als Zwischenprodukt z. B. Herbizide, Reaktivfarbstoffe, optische Aufheller und Additive für Polymere hergestellt werden. Cyanurchlorid ist an trockener Luft über den Siedepunkt hinaus beständig, sein chemisches Reaktionsverhalten entspricht etwa dem von Säurechloriden. Bei Kontakt mit Wasser kommt es zur Hydrolyse und Salzsäure $\left(\mathrm{HCI}_{(\mathrm{aq})}\right.$-Bildung. Es ist eine farblose, kristalline Substanz mit der Molmasse 184 und einem Schmelzpunkt von $146^{\circ} \mathrm{C}$ (Siedepunkt bei $194^{\circ} \mathrm{C}$ ). Cyanurchlorid wird in einer Reinheit von mindestens 99\% hergestellt. Die technische Herstellung von Cyanurchlorid ist in OECD SIDS (Organisation for Economic Co-operation and Development, Screening Information Data Sets) beschrieben [44].

Im Gegensatz $\mathrm{zu}$ anderen toxikologisch und epidemiologisch erforschten Stoffen liegen zu Cyanurchlorid keine für die Ableitung eines Grenzwerts belastbaren Tierstudien und auch keine umfangreichen Längsschnitt- bzw. Querschnitt-

Teil 2 dieses Beitrags finden Sie in Ausgabe $02 / 14$ von Zentralblatt für Arbeitsmedizin, Arbeitsschutz und Ergonomie; DOI 10.1007/ s40664-013-0002-4. 
studien am Menschen vor. In Beobachtungsstudien am Menschen wurden Reizwirkungen an Haut, Augen und Atemtrakt beschrieben und nach wiederholten Expositionen allergische Kontaktdermatiden [12, 29, 30, 39]. Allerdings ergeben diese epidemiologischen Arbeiten keine belastbaren Aussagen zu Dosis-Wirkungs-Beziehungen. Arbeitsmedizinisch sind Vorsorgeuntersuchungen nach G23 der berufsgenossenschaftlichen Grundsätze für cyanurchloridexponierte Arbeitnehmer vorgesehen [6].

Eine Grenzwertdiskussion kann derzeit im Wesentlichen nur auf Basis einer nicht voll belastbaren tierexperimentellen Studie geführt werden $[10,27,28]$. Andere subchronische oder chronische Inhalationsstudien liegen nicht vor [44]. Die tierexperimentelle Arbeit des Nofer Instituts in Lodz, Polen, beschreibt eine 90-Tage-Inhalationsstudie an Wistar-Ratten mit Cyanurchloridexpositionen von $0,01,0,05,0,25 \mathrm{mg} / \mathrm{m}^{3}$ sowie einer Kontrolle mit Umgebungsluft $[27,28]$. Die Autoren beschreiben insbesondere eine erhöhte Infektionsneigung bei Belastungen oberhalb von $0,05 \mathrm{mg} / \mathrm{m}^{3}$, was nach SIDS einen entsprechenden Grenzwert begründen könnte [44]. Allerdings handelt es sich um einen unpublizierten Forschungsbericht. Der Report der OECD merkt an, dass die Studie nicht nach guten Forschungspraxis (GLP, Good Laboratory Practice)-Bedingungen durchgeführt wurde [44] und die beschriebenen viralen Effekte sowohl in den exponierten Tieren als auch in den Kontrollen auftraten. Das Auftreten von Infekten unter den Kontrolltieren mindert die Belastbarkeit der Untersuchung erheblich, da die experimentellen Bedingungen somit vermutlich nicht den üblichen Standards entsprochen haben.

Da Cyanurchlorid aufgrund der hohen akuten inhalativen Toxizität nach Gefahrstoffverordnung mit $\mathrm{T}+$ (sehr giftig) bzw. entsprechend nach GHS (global harmonisiertes System, „globally harmonized system") mit Kategorie 2 und H 330 (Lebensgefahr beim Einatmen) gekennzeichnet werden muss [16], wird die Festlegung eines Arbeitsplatzgrenzwerts (AGW) zunehmend wichtiger.

Zur Erarbeitung eines Vorschlags für einen AGW wurden im Rahmen dieser
Studie vorhandene arbeitsmedizinische und Expositionsdaten zusammengestellt und aufbereitet. Zur epidemiologischen Zusammenhangsanalyse wird in Teil 2 berichtet [41].

\section{Material und Methoden}

\section{Kohorte}

Diese Untersuchung wurde als retrospektive Kohortenstudie angelegt. Die Studiengruppe besteht aus Mitarbeitern dreier cyanurchloridherstellender Werke der Evonik Industries AG (Antwerpen, Münchsmünster und Wesseling). Der Studienbeginn ist definiert als Produktionsbeginn des jeweiligen Werks. In Wesseling wird seit 1958 Cyanurchlorid produziert, in Münchsmünster erfolgte die Produktion im Zeitraum von 1977 bis 2009. In Münchsmünster handelt es sich um eine Freianlage, die sich in ihren Gegebenheiten - insbesondere im Hinblick auf die Exposition - von den in geschlossener Bauweise errichteten Anlagen in Antwerpen und Wesseling unterscheidet. In Antwerpen wurde von 1970 bis zur Schließung der Anlage Ende 2007 Cyanurchlorid produziert. Die Studiengruppe umfasst zwei Kategorien von Studienteilnehmern. Studienteilnehmer, die bis Ende 2007 in einem der drei Werke aktiv tätig waren, werden im Folgenden als aktive Studienteilnehmer bezeichnet. Studienteilnehmer, die vor Beginn der Studie ausgeschieden sind, aber in dem Zeitraum von Produktionsbeginn bis Studienbeginn in einem der drei Werke tätig waren, werden als historische Studienteilnehmer bezeichnet. Die Rekrutierung sowohl der aktiven als auch der historischen Mitarbeiter erfolgte durch Auswertung aller verfügbaren Personaldaten in den Personalabteilungen der Werke (z. B. aktuelle Personalakten oder Betriebstagebücher für die historischen Mitarbeiter).

Angestrebt wurde die Erfassung aller aktiven, männlichen Mitarbeiter Ende 2007 und aller ehemaligen, männlichen Mitarbeiter der Werke Antwerpen und Münchsmünster. Diese Kollektive stellen somit Berufsanfängerkohorten dar. Für das Werk Wesseling musste der Rekrutierungszeitraum der ehemaligen männlichen Mitarbeiter auf die Pe- riode 1974-2007 eingeschränkt werden, da keine Personalakten für den Zeitraum vor 1974 zur Verfügung standen. Dieses Kollektiv ergibt sich somit als Mischung aus einer Querschnitts- und einer Anfängerkohorte.

Das Studienkollektiv wurde weiter eingeschränkt auf männliche Studienteilnehmer, die in Summe länger als 12 Monate in einem Cyanurchloridbetrieb tätig waren. Das Follow-up der Studienteilnehmer lief bis Ende des Jahres 2007. Zu Beginn des Jahres 2008 wurden noch abschließende medizinische Untersuchungen nachgeholt. Das Studienende wird unabhängig davon im Folgenden als das Ende des Jahres 2007 berichtet.

\section{Cyanurchloridbelastung}

Die Höhe der Cyanurchloridbelastung ist in allen drei Werken orts-, zeit- und tätigkeitsabhängig. Grundsätzlich wird zwischen maskenpflichtigen und nichtmaskenpflichtigen Bereichen unterschieden. In den maskenpflichtigen Bereichen werden Vollmasken getragen. Im Rahmen einer Versuchsreihe wurden verschiedene alte und aktuelle Masken getestet. Es zeigte sich, dass die Cyanurchloridbelastung für einen Mitarbeiter unter Vollmaske immer unterhalb der Nachweisgrenze von Cyanurchlorid lag [35]. Der Bereich der Cyanurchloridabfüllung wurde in den Werken Antwerpen im Jahr 2004 und in Münchsmünster 1992 von maskenpflichtig auf nichtmaskenpflichtig umgestellt. Damit bestand in Antwerpen über die letzten 3 Jahre vor Studienende keine Maskenpflicht, in Münchsmünster wurden dagegen bereits über die letzten 15 Jahre vor dem Studienende keine Masken getragen.

Zur Abschätzung der Expositionshöhe standen Messprotokolle zu ortsbezogenen Messungen an verschiedenen Messorten und zu personengetragenen Messungen bei der Durchführung der unterschiedlichen Tätigkeiten zur Verfügung. Für diese Studie wurden zur Bestimmung des Cyanurchloridgehalts nur Messwerte berücksichtigt, die photometrisch (Messmethode „pH“) ermittelt, über Silicagelröhrchen nach NIOSH (National Institute for Occupational Safety and Health, Messmethode „NIOSH“) gemessen oder über einen Pro- 
Zbl Arbeitsmed 2014·64:10-25 DOI 10.1007/s40664-013-0001-5

(c) Springer-Verlag Berlin Heidelberg 2014

\section{P. Morfeld · B. Noll · W. Blumberg · R. Bauden · D. Strobl · E. Bock · U. Küpper · E. Gross · S. Schauhoff Cyanurchloridbelastung in Produktionsbetrieben. Teil 1: Kollektivbeschreibung, Expositionsdaten- aufbereitung sowie Erhebung medizinischer Response-Größen und wichtiger Kovariablen}

\section{Zusammenfassung}

Hintergrund. Cyanurchlorid $\left(\mathrm{C}_{3} \mathrm{Cl}_{3} \mathrm{~N}_{3}\right)$ ist ein weißes kristallines Pulver. Es wird für die Herstellung von Herbiziden benötigt, die in der Landwirtschaft eingesetzt werden. Weitere Verwendung findet es in der Produktion von Textilfarbstoffen und optischen Aufhellern oder der Herstellung von UV-Stabilisatoren für die Plastikindustrie sowie insbesondere für Automobilanwendungen in der Gummiindustrie. Zur Durchführung einer epidemiologischen Längsschnittstudie in drei Cyanurchloridproduktionsbetrieben wurde eine personenbezogene Expositionsermittlung durchgeführt sowie medizinische Response-Größen und wesentliche Kovariablen ermittelt.

Methoden. In den Betrieben Antwerpen, Münchsmünster und Wesseling der Evonik Industries AG wurden für alle länger als ein Jahr im Cyanurchloridbetrieb tätigen, männlichen, aktiven und ehemaligen Mitarbeiter zwischen 1958 und 2007 Cyanurchloridexpositionen, Arbeitsgeschichten, Angaben zum Rauchverhalten, biometrische Grunddaten, Lungenfunktionsparameter sowie Koexpositionen und Vorbelastungen aus vorangegangenen Tätigkeiten erfasst und auf Jahresdatensätze verdichtet. Zudem wurden Angaben zu chronischer Bronchitis, Atemnot, Asthma, chronisch-obstruktiver Bronchitis (COPD, , chronic obstructive pulmonary disease") und Sensibilisierungsparameter im Querschnitt erhoben. Für die Zuweisung von Expositionen zu personenbezogenen Tätigkeiten wurde eine Job-Exposure-Matrix (JEM) unter Verwendung von Konzentrationsmesswerten und unter Berücksichtigung des Tragens von Schutzmasken entwickelt. Um die Unsicherheit in den Belastungen aufgrund der schmalen Messdatenbasis besser abschätzen zu können, wurden über multiple Expositionsschätzungen 24 Varianten ermittelt.

Ergebnisse. Das Studienkollektiv umfasste 394 exponierte männliche Personen. Das durchschnittliche Alter lag bei 47,5 Jahren ( $\min .=21$, max. $=63, S D=10,3)$. Es gab 160 aktive Raucher, 78 Ex-Raucher und 149 Nie-Raucher. Der Mittelwert für die zum Zeitpunkt der letzten Lungenfunktionsuntersuchung aufgelaufenen Rauchmenge lag bei den aktiven Rauchern bei 18,9 Pack years $(\min .=0,3, \max .=69,5, S D=14,9$ ) und bei den Ex-Rauchern bei 19,4 Pack years (min.=1,2, max. $=105,0, S D=18,4)$. Die personenbezogene Durchschnittskonzentration der Cyanurchloridexposition betrug über alle Jahre im Mittel $0,019 \mathrm{mg} / \mathrm{m}^{3}(\mathrm{~min} .=0,003$, $\max .=0,044)$ bei einer Expositionsdauer von durchschnittlich 11,3 Jahren (min. $=0,58$, max.=34). Die kumulierte Gesamtexposition betrug durchschnittlich $0,217 \mathrm{mg} / \mathrm{m}^{3}$-Jahre $(\min .=0,005$, $\max .=0,941)$. Der Mittelwert der Cyanurchloridkonzentration pro Expositionsjahr lag bei $0,020 \mathrm{mg} / \mathrm{m}^{3}$ (min. $=0,003$, max. $=0,051$ ). Insgesamt standen 2983 Untersuchungen der Lungenfunktions- parameter VC (Vitalkapazität, ,"vital capacity"), FVC (forcierte Vitalkapazität, ,f forced vital capacity $^{\prime \prime}$ ) und FEV 1 (absolute Einsekundenkapazität, ,,forced expiratory volume in 1 second") zur Verfügung. Im Durchschnitt nahm jeder Studienteilnehmer an 7,6 medizinischen Untersuchungen mit Lungenfunktionsmessungen teil (min.=1, max.=19). Die VC in Prozent vom Sollwert betrug bei der ersten (letzten) Lungenfunktionsuntersuchung jedes Studienteilnehmers im Mittel 100\%, SD=13,6\% (103\%, SD=14,6\%). Die FVC in Prozent vom Sollwert lag im Mittel bei $103 \%, \mathrm{SD}=14,2 \%$ (103\%, SD = 15,4\%). Die FEV in Prozent vom Sollwert betrug im Mittel 99\%, $\mathrm{SD}=15,4 \%$ (99\%, SD $=18,3 \%$ ). Die FEV $1 \% F V C$ in Prozent vom Sollwert lag im Mittel bei 99\%, $\mathrm{SD}=10,4 \%$ (99\%, SD=12,2\%).

Diskussion. Die Studie stellt den weltweit umfassendsten Datenbestand zur Ermittlung der gesundheitlichen Auswirkung einer Langzeitexposition gegenüber Cyanurchlorid am Arbeitsplatz bereit. Bezogen auf externe Sollwerte ergaben die Lungenfunktionsmesswerte im Mittel keinen Hinweis auf Auffälligkeiten. Zur epidemiologischen Zusammenhangsanalyse wird in Morfeld u. Noll berichtet [41].

\section{Schlüsselwörter}

Cyanurchlorid - Expositionsermittlung . Job-exposure-Matrix · Epidemiologie · Arbeitsplatzbelastung

\section{Exposure to cyanuric chloride in production plants. Part 1: Cohort profile,} exposure assessment, medical data, and important covariables

\section{Abstract}

Purpose. Cyanuric chloride $\left(\mathrm{C}_{3} \mathrm{Cl}_{3} \mathrm{~N}_{3}\right)$ is a white crystalline powder. It is necessary for the production of herbicides used in agriculture. It is also used in the production of textile dyestuffs and optical brighteners or the manufacture of UV stabilizers for the plastics industry, and particularly for automotive applications in the rubber industry. As part of a long-term epidemiological study in three cyanuric chloride production facilities, we conducted an evaluation of the potential exposure to workers, collected medical data and key covariables.

Methods. In the Antwerp, Münchsmünster and Wesseling operations of Evonik Industries AG, we recorded cyanuric chloride measurement data, work histories, smoking behavior, basic biometric data, lung function parameters, as well as coexposure and prior exposure from past work of both active and former male employees who worked at least 1 year in the cyanuric chloride production between 1958 and 2007 and consolidated this information into annual data sets. Cross-sectional data on chronic bronchitis, shortness of breath, asthma, chronic obstructive pulmonary disease (COPD) and sensitization parameters were also collected. To assign exposure to jobs and tasks, we developed a job exposure matrix using concentration measurement values, and taking account of the wearing of protective masks. To estimate the uncertainty in exposure assessment based on the small number of measurement data, we constructed 24 variants by multiple exposure estimates.

Results. The cohort comprised 394 exposed male workers with an average age of 47.5 years (min. $=21, \max .=63, S D=10.3)$. There were 160 active smokers, 78 ex-smokers and 149 nonsmokers. The mean value for the quantity of smoke accrued at the time of the last lung function test was 18.9 pack-years for the active smokers ( $\min .=0.3, \max .=69.5, \mathrm{SD}=14.9)$ and 19.4 pack-years for the ex-smokers ( $\min .=1.2$, max. $=105.0, S D=18.4)$. Over all the years, the individual concentration of cyanuric chloride exposure was, on average, $0.019 \mathrm{mg} / \mathrm{m}^{3}$ ( $\min .=0.003$, max. $=0.044)$ given an exposure duration of, on average, 11.3 years $(\mathrm{min} .=0.58$, max.=34). The total cumulative exposure was, on average, $0.217 \mathrm{mg} / \mathrm{m}^{3}$-years $(\mathrm{min} .=0.005$, max. $=0.941$ ). The mean value of cyanuric chloride concentration per exposure year was $0.020 \mathrm{mg} / \mathrm{m}^{3}(\mathrm{~min} .=0.003$, max. $=0.051)$. A total of 2983 tests of the lung function parameters VC (vital capacity), FVC (forced vital capacity) and $\mathrm{FEV}_{1}$ (forced expiratory volume in 1 second) were available. On average, each study participant took part in 7.6 medical examinations with lung function measurements ( $\min .=1, \max .=19)$. As a percentage of the reference value, the $\mathrm{VC}$ was on average at $100 \%$, $S D=13.6 \%(103 \%, S D=14.6 \%)$ at the first (last) lung function test of each study participant, the FVC at $103 \%, S D=14.2 \%(103 \%, S D=15.4 \%)$ and the $\mathrm{FEV}_{1}$ at $99 \%, \mathrm{SD}=15.4 \%(99 \%, \mathrm{SD}=18.3 \%)$. As a percentage of the reference value, the mean $\mathrm{FEV}_{1} \% \mathrm{FVC}$ was at $99 \%, \mathrm{SD}=10.4 \%(99 \%$, $\mathrm{SD}=12.2 \%$ ).

Discussion. The study provides the most comprehensive pool of data worldwide for determining the health impact of long-term exposure to cyanuric chloride in the workplace. With regard to external reference values, the lung function measurement values, on average, reveal no abnormalities. For the epidemiological exposure response analysis, see Morfeld and Noll 2014 [41].

\section{Keywords}

Cyanuric chloride - Exposure assessment . Job exposure matrix Epidemiology . Occupational exposure 
Tab. 1 Verteilung der Jobkategorien pro SEG und Messort

\begin{tabular}{|c|c|c|c|c|c|c|}
\hline SEG, Messort & $\begin{array}{l}\text { Mess- } \\
\text { warten- } \\
\text { fahrer }\end{array}$ & $\begin{array}{l}\text { An- } \\
\text { lagen- } \\
\text { fahrer }\end{array}$ & $\begin{array}{l}\text { Abfüller } \\
\text { mit } \\
\text { Maske }\end{array}$ & $\begin{array}{l}\text { Abfüller } \\
\text { ohne } \\
\text { Maske }\end{array}$ & $\begin{array}{l}\text { Versand- } \\
\text { arbeiter }\end{array}$ & $\begin{array}{l}\text { Labo- } \\
\text { rant }\end{array}$ \\
\hline Messwarte & $P$ & $P, A, L$ & A & A & & \\
\hline Messwarte Aufenthaltsraum & $\mathrm{P}$ & $P, A, L$ & $P, A$ & $P, A$ & V & $\mathrm{L}$ \\
\hline Anlage maskenpflichtig & & $P, A, L$ & & & & \\
\hline Anlage nicht maskenpflichtig & $\mathrm{P}$ & $P, A, L$ & & & & \\
\hline Abfüllung mit Maske & $P$ & $\mathrm{P}$ & $P, A$ & & & \\
\hline Abfüllung ohne Maske & $P$ & $P$ & & $P, A$ & & \\
\hline Abfüllung Aufenthaltsraum & & & A & $A$ & V & \\
\hline Lager & & $\mathrm{P}$ & A & A & V & \\
\hline Labor & & $P$ & & & & $\mathrm{~L}$ \\
\hline Sieblabor & & $P$ & & & & \\
\hline \multicolumn{7}{|c|}{$\begin{array}{l}\text { Diese Aufteilung wurde zusätzlich nach Werken differenziert (nicht dargestellt).P Produktionsmitarbeiter, } A \text { Aus- } \\
\text { tragsmitarbeiter, } L \text { Laborant, } V \text { Versandmitarbeiter, SEG, similar exposure group“ (Expositionsgruppe ungefähr } \\
\text { gleicher Tätigkeit, gleicher Expositionsart und -belastung). }\end{array}$} \\
\hline
\end{tabular}

benahmekopf GGP (Gesamtstaub-GasProbenahme) nach BIA (Berufsgenossenschaftliches Institut für Arbeitsschutz, Messmethode „BIA_GGP_3040“) erhoben wurden. Bei der Messmethode " $\mathrm{pH}^{\text {“ }}$ handelt es sich um eine ältere Messmethode für gasförmiges Cyanurchlorid, die zwischen 1979 und 1987 in Wesseling zum Einsatz kam. Es wurden nur ortsbezogene Messungen durchgeführt. Der Cyanurchloridgehalt wurde spektralphotometrisch nach Impinger-Probenahme in einer Absorptionslösung bestimmt [42]. Mit der Messmethode „NIOSH“ für gasförmiges Cyanurchlorid wurden sowohl ortsbezogene als auch personengetragene Messungen in allen Werken in den Jahren 1992 bis 2005 durchgeführt. Die Probenahme erfolgte durch Adsorption auf Silicagel-Röhrchen [33]. Bei der Messmethode „BIA_GGP_3040" handelt es sich um eine kombinierte Staub- und Gasprobenahme, die in allen Werken zwischen 2005 und 2007 sowohl für ortsbezogene als auch für personengetragene Messungen zum Einsatz kam. Mithilfe einer Probenahmepumpe wird ein definiertes Luftvolumen über einen BIA-Probenahmekopf GGP gesaugt [1]. Die Analyse des Cyanurchloridgehalts einer Probe erfolgte per Hochleistungsflüssigkeitschromatographie (HPLC-Methode, „high-performance liquid chromatography", [43]), spektralphotometrisch [42] oder per Fourier-Transform-Infrarotspektrometer (FTIR)-Spektroskopie [3]. Zusätzlich berücksichtigt wurde der Messwert aus einer Staubmessung, deren Glasfaserfil- ter auf Cyanurchlorid untersuchte wurde. Da zu diesem Zeitpunkt die Messmethode "NIOSH“ das gängige Messverfahren war, wurde dieser unterhalb der Nachweisgrenze liegende Messwert dieser Messmethode zugeordnet.

Die Messdaten der verschiedenen Werke wurden einheitlich aufbereitet und in einem Teildatenkörper Messdaten zusammengefasst. Zur Behandlung von Messwerten unterhalb der Nachweisgrenzen und bei Verwendung einer Schutzmaske siehe Abschn. Aufbereitung der Cyanurchloridmessdaten.

\section{Rotationen}

Durch Rotation zwischen verschiedenen - andere chemikalienproduzierenden Bereichen war ein großer Teil der Studienteilnehmer nur in begrenzten Zeitabschnitten einer Cyanurchloridbelastung ausgesetzt. In den Werken Antwerpen und Wesseling wurde zwischen den Produktionsbereichen für Cyanurchlorid und für Salzsäure $\left(\mathrm{HCl}_{(\mathrm{aq})}\right)$ rotiert, während im Werk Münchsmünster eine Rotation zwischen den Produktionsbereichen für Kalziumchlorid $\left(\mathrm{CaCl}_{2}\right)$, Natriumdicyanamid $\left(\mathrm{C}_{2} \mathrm{~N}_{3} \mathrm{Na}, \mathrm{NDC}\right)$, Acetoncyanhydrin $\left(\mathrm{C}_{4} \mathrm{H}_{7} \mathrm{NO}, \mathrm{ACH}\right)$ und Cyanwasserstoff ( $\mathrm{HCN}$ ) stattfand. In der NDC-Anlage sind die Mitarbeiter exponiert gegenüber NDC-Staub (Cyanamid, $\mathrm{CH}_{2} \mathrm{~N}_{2}$, und Chlorcyan, $\mathrm{CClN}$ ), Methanol $\left(\mathrm{CH}_{4} \mathrm{O}\right)$ und Chlormethan $\left(\mathrm{CH}_{3} \mathrm{Cl}\right)$. Eine $\mathrm{HCl}_{(\mathrm{aq})}$-Rotation gab es im Werk Münchsmünster nicht, stattdes- sen wurde $\mathrm{HCl}_{(\mathrm{aq})}$ als Koexposition für 117 von 120 Studienteilnehmern im Werk Münchsmünster ermittelt. Diese Chemikalien wurden nach Expertenurteil (Koautor Ulrich Küpper) entsprechend ihrer erwarteten Auswirkung auf die Lungenfunktion in zwei Gruppen geordnet. Die erste Gruppe besteht aus HCN, ACH und den in der NDC-Anlage vorhandenen Expositionen. Hier sind keine Lungenfunktionsveränderungen beschrieben und somit keine Auswirkungen auf die Lungenfunktion zu erwarten. Diese Gruppe wurde daher nicht als Confounder betrachtet. Die zweite Gruppe bilden die Chemikalien $\mathrm{CaCl}_{2}$ und $\mathrm{HCl}_{\text {(aq) }}$. Sie haben eine starke Reizwirkung auf die Atemwege und könnten (bei entsprechender Exposition) ebenso wie Cyanurchlorid zu Veränderungen der Lungenfunktion führen, sodass diese Belastungen als potenzieller Confounder eingestuft wurden.

\section{Entwicklung einer JEM}

Voraussetzung für die Entwicklung einer homogenen JEM [23]ist die Erstellung eines werksübergreifend anwendbaren Tätigkeitsprofils für alle exponierten Mitarbeiter. Hierzu wurden Jobkategorien harmonisiert und Messorte und Tätigkeiten soweit möglich unter einheitlichen Bezeichnungen zusammengefasst. Über Expertenschätzungen erfolgte anschlieBend die Aufteilung der Jobkategorien in prozentuale Zeitanteile für Messorte und Tätigkeiten. Es wurden vier Jobkategorien, zehn Messorte und sechs Tätigkeitsprofile zur Bildung von Expositionsgruppen ungefähr gleicher Tätigkeit, gleicher Expositionsart und -belastung (SEG, „similar exposure group“) auch unter Berücksichtigung des Einsatzes von Schutzmasken definiert. $\bullet$ Tab. 1 stellt die Verteilung der Jobkategorien pro SEG und Messort dar.

Damit konnten die Konzentrationshöhen von Cyanurchlorid aus dem Teildatenkörper Messdaten an die entsprechenden Jobkategorien und damit an die relevanten SEGs und Messorte angebunden werden. Der so erstellte Teildatenkörper JEM beschreibt die Expositionsbelastung durch Cyanurchlorid in Abhängigkeit von der betreffenden Jobkategorie 
und den darüber zugeordneten vergleichbaren SEGs und Messorten.

Durch Rotation zwischen verschiedenen - andere chemikalienproduzierenden - Betrieben waren Studienteilnehmer nur in begrenzten Zeitabschnitten einer Cyanurchloridbelastung ausgesetzt, was bei der JEM-Entwicklung berücksichtigt wurde.

\section{Erfassung personenbezogener Daten unter Einhaltung der Datenschutzkriterien}

Alle im Rahmen dieser Studie relevanten personenbezogenen Daten wurden über eine speziell zu diesem Zweck entwickelte Software erfasst, der sog. Health Study Application (HSA). In dieser Software wird eine sog. Pseudonymisierungsliste geführt, auf die nur der Datenschutzbeauftragte als Vertrauensstelle für diese Studie Zugriff hatte. Sie enthält eine Pseudonymisierungs-ID (PID) und die dazugehörigen Personenidentifikationsmerkmale. Ein entsprechendes Berechtigungskonzept stellt sicher, dass nur das mit der Erhebung vor Ort befasste Personal die Personenidentifikationsmerkmale sieht und hierzu Daten erfasst. Ein Zugriff besteht ausschließlich auf die Datenbestände, die in der eigenen Verantwortung liegen. Die Daten werden über die Anwendung verschlüsselt gespeichert und zur epidemiologischen Auswertung über die PID pseudonymisiert aus der Software als Teildatenkörper HSA exportiert. Die Software beinhaltet einen Rückmeldeprozess für die Abstimmung von Fehlern bzw. Unstimmigkeiten bei der Datenerfassung zwischen Erfassern und Epidemiologen bzw. dem Datenmanagement, sodass Rückfragen zu offenen Daten unterbunden waren.

\section{Erfassung der Tätigkeiten}

Zur Erstellung der Tätigkeitsprofile der Studienteilnehmer wurden sowohl aus den Personalakten der Personalabteilung als auch aus den medizinischen Akten des arbeitsmedizinischen Dienstes alle Tätigkeiten eines aktiven bzw. historischen Studienteilnehmers erfasst. Hierbei wurden die Personalakten der Personalabteilung per Definition als führendes System betrachtet, das bei fehlenden Einträgen um
Daten aus der arbeitsmedizinischen Akte (sofern dort vorhanden) ergänzt wurde. Dokumentiert wurden Beginn und Ende einer Tätigkeit mit entsprechendem Beginn- bzw. Enddatum, Tätigkeitsart und Betrieb, in dem die Tätigkeit ausgeführt wurde, sowie eventuell vorhandene Vorbelastungen der Studienteilnehmer, die in den aktuellen Personalakten der aktiven Studienteilnehmer bzw. in den Personalakten oder Betriebstagebüchern der historischen Studienteilnehmer erfasst waren. Die Tätigkeiten wurden so detailliert erhoben, dass sie eindeutig den SEGs zugeordnet werden konnten. Die in den Personalakten oder Betriebstagebüchern dokumentierten Vorbelastungen der Studienteilnehmer vor Beginn einer Beschäftigung in einem Cyanurchloridbetrieb, die auf eine Belastung der Lunge als Zielorgan hinweisen könnten wie Arbeiten in der Chemiebranche, als Maurer, Schlosser, Schreiner, Maler oder in einem Bergbaubetrieb, wurden gemäß der internationalen Standardklassifikation der Wirtschaftszweige (ISIC, International Standard Industrial Classification) kategorisiert [26].

Eine Erfassung von Tätigkeiten der Studienteilnehmer nach Ausscheiden aus dem Betrieb entfällt, da in dieser Studie medizinische Endpunkte analysiert wurden, die während der Tätigkeit im Cyanurchloridbetrieb erhoben wurden (z. B. Lungenfunktionsdaten). Das Follow-up dieser Studie endet vor oder bei Verlassen des Cyanurchloridbetriebs.

\section{Erfassung der medizinischen Daten und Kovariablen}

Die zentrale Zielgröße dieser Studie war die Lungenfunktion. Zur Erhebung der medizinischen Befunde wurden neben Daten zur Lungenfunktion auch Alter, Herkunftsland, biometrische Grunddaten wie Körpergewicht und Körpergröße aufgezeichnet. Das Rauchverhalten wurde durch Angabe von Raucherstatus (NieRaucher, Ex-Raucher, aktiver Raucher), Rauchmenge (Stückzahl pro Tag), Rauchdauer (in Jahren), Rauchbeginn und Rauchende (jeweils Datum) zeitabhängig dokumentiert.

Folgende Lungenfunktions-Parameter wurden in dieser Studie betrachtet:
- FVC bzw. bei glockenspirometrischen Messungen der VC: Die forcierte Vitalkapazität ist definiert als Volumendifferenz zwischen maximaler Ein- und Ausatmung [50].

- $\mathrm{FEV}_{1}$ : Die absolute Einsekundenkapazität - auch Atemstoß oder forcierte Einsekundenkapazität - bezeichnet das Atemvolumen, welches der Proband bei der forcierten Ausatmung innerhalb der ersten Sekunde auszuatmen vermag [50].

- Relative Einsekundenkapazität (Tiffenau-Wert): berechnet als Quotient aus $\mathrm{FEV}_{1}$ und $\mathrm{FVC}\left(\mathrm{FEV}_{1} \% \mathrm{FVC}\right.$, [50]).

Während im Werk Antwerpen pro Lungenfunktionsuntersuchung nur das jeweils beste Ergebnis aus drei Lungenfunktionsmessungen dokumentiert wurde, standen für die Werke Münchsmünster und Wesseling alle bei einer Untersuchung durchgeführten Lungenfunktionsmessungen zur Verfügung. In allen Werken wurden die Lungenfunktionsparameter entsprechend den Kriterien nach Goldstandard erhoben [18, 19].

Zum externen Vergleich wurden die Lungenfunktionssollwerte nach Quanjer et al. herangezogen [46]. Als weitere Referenzwerte berechneten wir die Lungenfunktionssollwerte nach der globalen Lungeninitiative (GLI, Global Lungs Initiative) mit einem „Excel sheet calculator", der auf der Internetseite der GLI zur Verfügung gestellt wurde [17].

Im Studienzeitraum 1958-2007 wurden insgesamt 4 verschiedene Lungenfunktionsmesssysteme für die Ermittlung der Lungenfunktionsparameter eingesetzt (Glockenspirometer [4], Vitalograph [52, 54], CustoVit [45, 51], Bodyplethysmographie $[15,51])$.

Um potenzielle Verzerrungen in der Analyse zu minimieren, wurde der Einsatz der verschiedenen Lungenfunktionsmessgeräte zeit- und personenabhängig festgehalten.

Aus Anlass der Studie fand für die aktiven Studienteilnehmer eine zusätzliche medizinische Untersuchung statt, in der neben einer weiteren Lungenfunktionsuntersuchung für 130 Studienteilnehmer zusätzlich eine Blutuntersuchung zur Bestimmung von Sensibilisierungspara- 


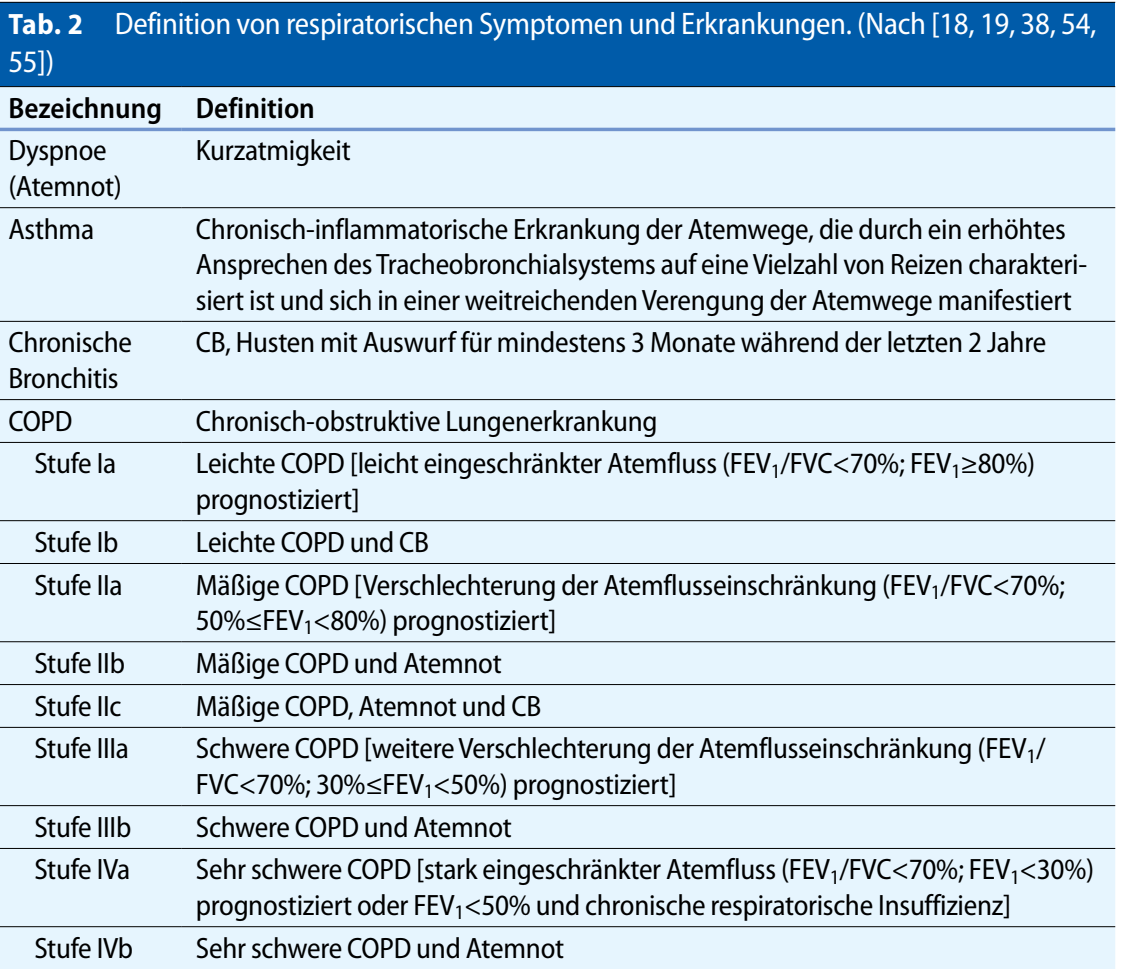

metern durchgeführt wurde und 157 Studienteilnehmer einen Fragebogen zu Atemwegserkrankungen und Rauchgewohnheiten ausfüllten.

Alle aktiven Mitarbeiter wurden über den Zweck der Untersuchung aufgeklärt. Die Teilnahme war freiwillig. Alle Teilnehmer haben der Untersuchung und der Auswertung der Daten in Form einer Einverständniserklärung schriftlich zugestimmt.

Aus den Fragebogenangaben wurden Indikatoren für Atemwegserkrankungen abgeleitet. Bestimmt wurden: das Vorliegen von Atemnot (Kurzatmigkeit), das Vorliegen von Asthma [38, 55], das Vorliegen von chronischer Bronchitis (Husten mit Auswurf über mindestens 3 Monate während der letzten beiden Jahre, [54]) und in Kombination mit Lungenfunktionsmesswerten das Vorliegen verschiedener Grade der COPD [18, 19]. Aus den Fragebogenantworten wurde der Asthmaindikator gebildet: Der Asthmaindikator wurde auf $J a$ gesetzt, wenn entweder ohne Vorliegen einer Erkältung ein manchmal keuchendes bzw. pfeifendes Atemgeräusch angegeben wurde (1) oder bereits ein Asthmaanfall vorlag (2). Auch wenn Asthma bereits von einem Arzt diagnostiziert wurde und eine medizinische
Behandlung des Asthmas erfolgt ist (3), wurde der Indikator auf $J a$ gesetzt. Der Asthma-Indikator wurde auf Nein gesetzt, wenn (1), (2) und (3) mit Nein beantwortet wurden. Chronische Bronchitis (CB) wurde wie folgt operationalisiert: Der CB-Indikator wurde auf $J a$ gesetzt, wenn ein dauerhafter Husten mit begleitendem Auswurf seit mehr als 3 aufeinanderfolgenden Monaten angegeben wurde (1) und diese Symptome seit mehr als 2 Jahren anhielten (2). Der CB-Indikator wurde auf Nein gesetzt, wenn (1) oder (2) mit Nein beantwortet wurden. Die Grade der COPD wurden definiert wie in $\bullet$ Tab. 2 beschrieben.

Als Sensibilisierungsparameter wurden im Blutserum das Gesamt-Immungobulin-E (IgE) und das spezifische IgE bestimmt $[31,32]$. Die Serumproben wurden eingefroren und dem akkreditierten Labor der Currenta GmbH \& Co. OHG (ehemals: Bayer Industry Services GmbH \& Co. OHG) unter der Leitung von Prof. G. Leng zur Analyse zugestellt [47]. Als Nachweisgrenzen des Verfahrens (Fluoreszenz-Enzym-Immunoassay, FEIA) gelten für Gesamt-IgE 0,2 kU/l bzw. für spezifisches IgE 0,1 kU/l; als Referenzgrenzen des Normalbereichs gelten 300 bzw. $0,35 \mathrm{kU} / 1[24,25]$. Messwerte unterhalb der Nachweisgrenze wurden auf den halben Wert der jeweiligen Nachweisgrenze gesetzt.

\section{Datenaufbereitung}

\section{Aufbereitung der Cyanurchlorid-Messdaten}

Für Cyanurchlorid waren für alle 3 Werke insgesamt 196 Messungen dokumentiert. Messungen mit nicht klar definierten bzw. dokumentierten Messmethoden und Messumständen wurden verworfen. Es standen insgesamt 188 verwertbare Messwerte (Antwerpen: $n=52$, Münchsmünster: $n=31$, Wesseling: $n=105$ ) für die Messdatenmodellierung zur Verfügung.

Für diese Studie wurde in den maskenpflichtigen Bereichen aller Werke ein Maskenwert angesetzt, der in einem Gasmaskentest aus Anlass dieser Studie unter Anwendung der Messmethode "NIOSH“ als Belastungswert hinter der Schutzmaske ermittelt wurde [35]. In den Werksbereichen, in denen das Tragen einer Vollgesichtsfiltermaske vorgeschrieben ist, war zu Beginn der Studie unklar, welcher Cyanurchloridexposition ein Studienteilnehmer hinter dem Maskenfilter der Vollgesichtsfiltermaske ausgesetzt ist. Ziel des von Kunz und Groß [35] beschriebenen Gasmaskentests war eine praxisnahe Ermittlung der Expositionskonzentration hinter dem Maskenfilter einer Vollgesichtsmaske für die in den Betrieben über die jeweilige Betriebslaufzeit verwendeten Masken und Maskenfilter. Ein wesentliches Ergebnis des Gasmaskentests war, dass hinter dem Maskenfilter keine Cyanurchloridkonzentrationen oberhalb der Verfahrensnachweisgrenze von $0,003 \mathrm{mg} / \mathrm{m}^{3} \mathrm{zu}$ messen waren. Für alle Werke und alle relevanten Filtertypen wurde aus diesem Grund ein Maskenwert von $0,003 \mathrm{mg} / \mathrm{m}^{3}$ festgelegt. Von den 188 Messwerten wurden somit die in den maskenpflichtigen Bereichen gemessenen Werte nicht berücksichtigt, sondern stattdessen der Maskenwert angesetzt $(n=64)$. Durch Rückfragen in den Werken (Betriebsleitung, Arbeitsschutz, Arbeitsmedizin, Betriebsrat, Mitarbeiter) wurde sichergestellt, dass von den Mitarbeitern in den maskenpflichtigen Bereichen immer Gasmasken getragen wurden. Dies wird aufgrund der Reizwirkung des Cya- 


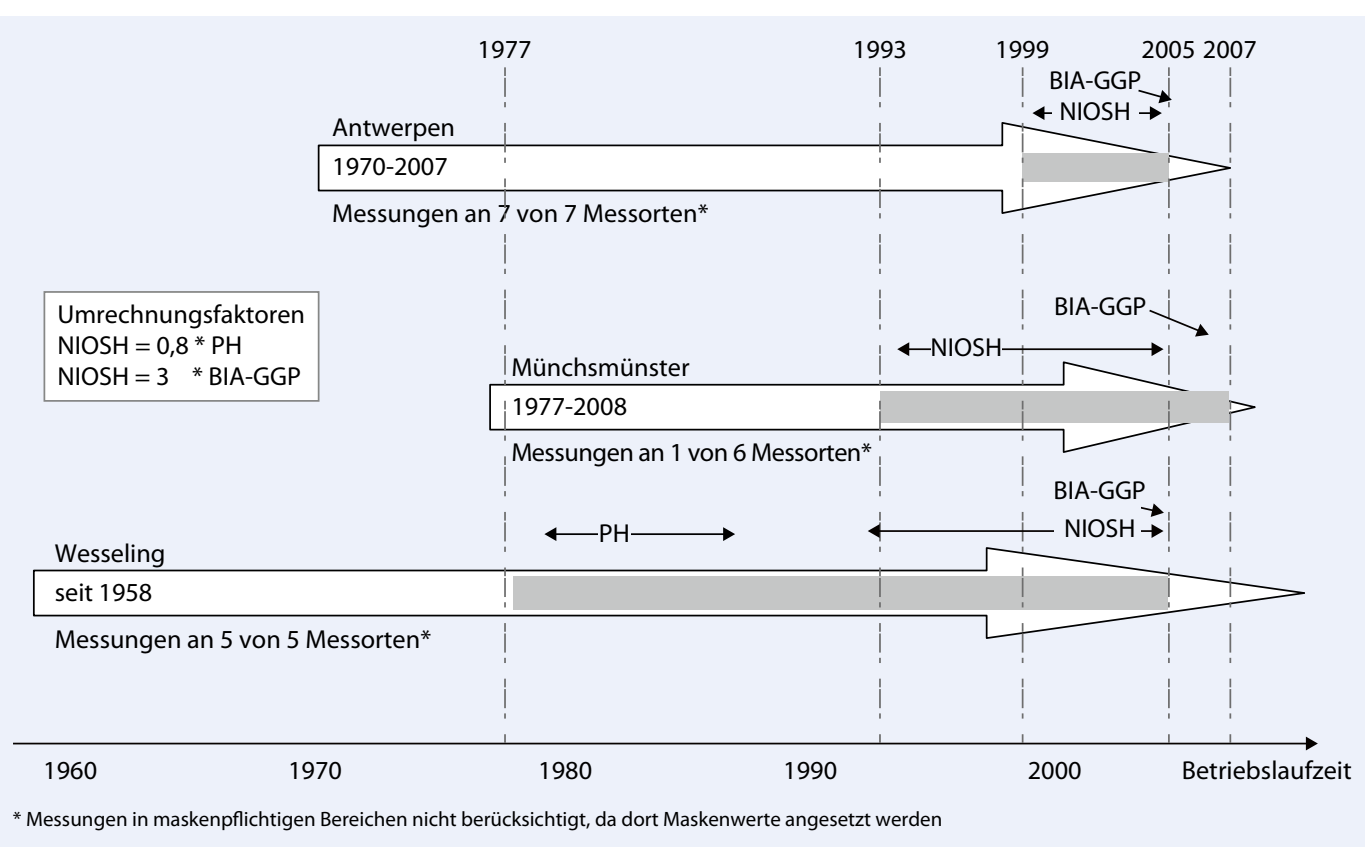

Abb. $1<$ Messdatenabdeckung und Messmethoden nurchlorids auf die Schleimhäute schon im eigenen Interesse von den Mitarbeitern beachtet. Es war auch das besondere Interesse des Betriebsrats, alle Mitarbeiter vor den unangenehmen und potenziell gesundheitsgefährdenden Expositionen zu schützen. Über eine Standardprozedur überprüft der Mitarbeiter die Dichtigkeit der Maske vor dem Einsatz in einem maskenpflichtigen Bereich. Eine Undichtigkeit würde recht schnell bemerkt und $\mathrm{zu}$ einem Austausch der Gasmaske führen.

Für Messwerte in nichtmaskenpflichtigen Bereichen und oberhalb der jeweiligen Nachweisgrenze ( $n=67)$ wurden Umrechnungsfaktoren für die Normierung von Messmethode („BIA_GGP_3040“, „pH“, „NIOSH“) und Messart (personenbezogen, ortsbezogen) mithilfe multipler linearer Regressionsmodelle ermittelt. Der Logarithmus der Messwerte oberhalb der Nachweisgrenze wurde hierzu in Abhängigkeit von Messmethode und Messart sowie der Kombination aus Werk-Messort-SEG gefittet (ggf. mit Mehrfachzuordnung der Messwerte zu SEGs). Die Berücksichtigung der Kombination Werk-Messort-SEG als Kovariable adjustiert die geschätzten Umrechnungsfaktoren für Unterschiede in dieser Kombination. Das Modell wurde im Sinne einer Sensitivitätsanalyse zudem a) exakt auf die Messdaten (keine Mehrfachzuordnung) beschränkt und b) um das Messjahr als Kovariable erweitert, um für zeitliche Unterschiede zu adjustieren.

Der interne Vergleich der Messdaten zur Normierung der Messmethoden auf „NIOSH“ ergab einen Faktor von 3 für die Umrechnung der Messmethode „BIA_ GGP_3040“ nach „NIOSH“ und einen Faktor von 0,8 für die Umrechnung der Messmethode „pH“ nach „NIOSH“. Für die Normierung der Messart auf personengetragen ergab der interne Vergleich der Messdaten einen Umrechnungsfaktor von 1; hier ergab die Analyse also, dass bei der Entwicklung der Expositionsschätzer nicht zwischen personen- und ortsbezogenen Messungen unterschieden werden muss. Bis auf die Umrechnung der Messmethode von „pH“ nach „NIOSH“ erwiesen sich die Umrechnungsfaktoren als relativ stabil. Die ältere Messmethode „pH“ kam nur in Wesseling in den Jahren 19791987 zum Einsatz, wodurch eine werksübergreifende Adjustierung nicht möglich war. Zudem gab es keine zeitliche Überlappung der Messmethoden „pH“ und „NIOSH“, da „NIOSH“ frühestens ab 1992 verwendet wurde. Dies erklärt die Unsicherheit der Schätzung dieses Umrechnungsfaktors. In einem internen Ringversuch der Evonik Industries AG wurde allerdings ebenfalls ein Umrechnungsfaktor von „pH“ nach „NIOSH“ von ca. 0,8 ermittelt [42], der daher den auf Basis der Messdaten mit den Regressionsmodellen geschätzten Umrechnungsfaktor stützt. Deshalb wurden beide Umrechnungsfaktoren („BIA_GGP_3040“ nach „NIOSH“: Faktor=3, „pH“ nach „NIOSH“: Faktor $=0,8$ ) in den weiteren Abschätzungen der Expositionshöhen verwendet. - Abb. 1 gibt einen Überblick zur Messdatenabdeckung und Messmethode über die Betriebslaufzeiten der 3 Werke.

Für Messwerte in nichtmaskenpflichtigen Bereichen und unterhalb der Nachweisgrenze $(n=57)$ wurden drei verschiedene Imputationsverfahren verwendet. Die Messwerte wurden a) auf den Wert der Nachweisgrenze (MWG=NWG/1) gesetzt (Verfahren "nwgl“) und alternativ b) auf die halbe Nachweisgrenze NWG/2 (Verfahren „nwg2“) bzw. c) auf NWG/ $\sqrt{2}$ (Verfahren „nwgsqr2“). Zu diesen Varianten des Umgangs mit Messwerten unterhalb einer Nachweisgrenze siehe die Ausführungen von Hornung und Reed [22].

Da der Umfang der Messwerte beschränkt war, nicht alle Zeiten gleichmäßig mit Messungen überdeckt und auch nicht immer dieselben Messverfahren eingesetzt wurden, haben wir die Expositionen unter variierenden Annahmen mehrfach eingeschätzt, um die innewohnenden Unsicherheiten abzubilden. In diesen multiplen Expositionsschätzungen wurden alle Messwerte oberhalb und unterhalb der Nachweisgrenze berück- 
Tab. 3 Varianten der multiplen Expositionsschätzung

\begin{tabular}{|c|c|c|c|}
\hline \multirow{2}{*}{$\begin{array}{l}\text { Variantenbildung in der Mess- } \\
\text { datenverarbeitung (ohne } \\
\text { - und mit + Umrechnung von } \\
\text { Messmethode und Messart) }\end{array}$} & \multicolumn{3}{|c|}{$\begin{array}{l}\text { Bezeichnung der Messwerte nach der Art der Setzung von } \\
\text { Messwerten } \leq \text { NWG }\end{array}$} \\
\hline & 1. Ansatz auf NWG & $\begin{array}{l}\text { 2. Ansatz auf } \\
\text { NWG/2 }\end{array}$ & $\begin{array}{l}\text { 3. Ansatz }{ }^{\mathrm{a}} \text { auf } \\
\mathrm{NWG} / \sqrt{ } 2\end{array}$ \\
\hline Mittelwert - & mw_nwg1 & mw_nwg2 & mw_nwgsqr2 \\
\hline Mittelwert + & mw_nwg1_umr & mw_nwg2_umr & mw_nwgsqr2_umr \\
\hline 1. Quartil - & p25_nwg1 & p25_nwg2 & p25_nwgsqr2 \\
\hline 1. Quartil + & p25_nwg1_umr & p25_nwg2_umr & p25_nwgsqr2_umr \\
\hline 2. Quartil - & p50_nwg1 & p50_nwg2 & p50_nwgsqr2 \\
\hline 2. Quartil + & p50_nwg1_umr & p50_nwg2_umr & p50_nwgsqr2_umr \\
\hline 3. Quartil - & p75_nwg1 & p75_nwg2 & p75_nwgsqr2 \\
\hline 3. Quartil + & p75_nwg1_umr & p75_nwg2_umr & p75_nwgsqr2_umr \\
\hline
\end{tabular}

sichtigt, die nicht durch den Maskenwert überschrieben wurden.

Die Häufigkeitsverteilung der Messwerte zu einer interessierenden Kombination (z. B. Werk-Messort-SEG) kann in verschiedener Weise durch Statistiken zusammengefasst werden. Oft wird allein auf den Mittelwert oder allein auf den Median der Messwerte verdichtet. Allerdings könnten die Ergebnisse der Studie davon abhängen, welche Statistik zur Zusammenfassung der Messwerte verwendet wird. Deshalb wurde in dieser Studie sowohl der arithmetische Mittelwert als auch alternativ der Median (p50=50. Perzentil) zur Verdichtung verwendet. Um die Unsicherheit der Messwerte vollständiger in den Auswertungen abbilden zu können, wurden zudem die 2. und 4. Quartile (p25=25. Perzentil, p75=75. Perzentil) der Häufigkeitsverteilungen als Stützstellen verwendet.

Insgesamt ergaben sich damit für die multiplen Expositionsschätzungen $2 \times 3 \times 4=24$ Varianten zur Ermittlung der Cyanurchloridexposition in dieser Studie (- Tab. 3). Eine parallele Durchführung der epidemiologischen Analysen zu diesen 24 Varianten der Expositionsschätzung erlaubt zu beurteilen, ob die Ergebnisse relevant davon abhängen, welche Schätzung verwendet wird. Eine solche Sensitivitätsanalyse gibt also Einblick, ob die Messwerte zu unsicher sind, um eine robuste Schlussfolgerung zu erlauben.

\section{Imputation der Belastungshöhen gegenüber Cyanurchlorid bei fehlenden Daten}

Epidemiologische Studien zu Themen aus der Arbeitswelt leiden häufig unter einer unzureichenden Expositionsabschätzung [7].

Eine geringe Anzahl an, wie in dieser Studie, verwertbaren Messdaten generiert eine unvollständige Datenmatrix, in der die vorhandenen Messwerte nicht ausreichen, um die Expositionsbelastung der Mitarbeiter tätigkeitsabhängig über den gesamten Studienzeitraum zu beschreiben [48]. Üblicherweise sinken die Konzentrationen an den Arbeitsplätzen in ausgeprägter Weise mit der Zeit [8]. Eine geringe und auf einen aktuelleren Zeitraum beschränkte Messdatenabdeckung kann daher zu einer Missklassifikation in der Expositionsschätzung führen, wenn die Expositionsbewertung allein auf die vorhandenen Konzentrationsmesswerte gestützt wird [34]. Daraus resultieren potenziell verzerrte Risikoschätzungen in der epidemiologischen Analyse [9]. In einer solchen Situation ist es somit nicht angemessen, Expositionsschätzungen allein auf die im Wesentlichen aktuelleren Messwerte der Konzentrationen zu stützen [7, 36]. Wesentliche epidemiologische Projekte [5, 2, 49], die eine zentrale Rolle in der nationalen und internationalen Karzinogenitätseinstufung von beruflicher Formaldehydexposition oder von Belastungen mit Dieselmotoremissionen einnehmen, folgten dieser methodischen Vorgabe.

Um die oben beschriebenen Schwächen auszugleichen, wurde unter Berücksichtigung der zusammen mit Experten entwickelten Jobkategorien und SEGs [23] versucht, Expositionsschätzungen vorzunehmen, die bis zum Produktionsbeginn der einzelnen Werke zurückreichen, und in die sowohl die vorliegenden Messwer- te als auch Erfahrungen der Experten zur historischen Entwicklung der Produktions- und Belastungssituation eingehen [20].

In Bereichen ohne Messdateninformationen wurde zunächst versucht, die Messdatenhistorie über Expertenwissen abzubilden und hierdurch die Lücken zwischen den vorhandenen Messwerten aufzufüllen. Während in Antwerpen und Wesseling zu allen Messorten mindestens ein Messwert vorlag, gab es in Münchsmünster nur Messungen zu dem Messort Abfüllung ohne Maske. Für alle anderen Messorte in Münchsmünster wurden die Modellschätzer für Antwerpen und Wesseling unter zusätzlicher Berücksichtigung von Expertenwissen imputiert.

Für die Modellierung der fehlenden Messdaten wurde in Abstimmung mit den Experten Geruchsschwelle bzw. Maskenpflichtschwelle für Cyanurchlorid herangezogen. Der Geruchsschwellenwert wurde im Mittel mit $0,0385 \mathrm{mg} / \mathrm{m}^{3}$ (min.=0,007, $\max .=0,07)$ angesetzt [37]. Als Maskenpflichtschwellenwert wurde ein interner Erfahrungswert von Betrieben der Evonik Industries AG angesetzt, der dem Doppelten des Geruchsschwellenwerts entspricht und damit im Mittel $0,077 \mathrm{mg} / \mathrm{m}^{3}$ beträgt ( $\min .=0,014$, $\max$. $=0,14$ ).

Insbesondere $\mathrm{zu}$ Produktionsbeginn lagen für alle Werke keine Messdaten vor, sodass hier die Konzentrationsdaten rückwärts extrapoliert wurden. Diese Rückwärtsextrapolation wurde an den im Wesentlichen aktuelleren Messdaten verankert, wobei mehrere solcher Ausgangspunkte zur Abbildung der Unsicherheit der Messdatenlage angesetzt wurden.

Zur Veranschaulichung einer solchen Rückwärtsextrapolation mithilfe von Regressionsrechnungen und unter Berücksichtigung von Expertenwissen wird das Beispiel eines Produktionsmitarbeiters im Werk Wesseling im Bereich der nicht maskenpflichtigen Anlage gewählt. Insgesamt lagen 27 verwertbare Messungen aus folgenden Jahren vor: $1979(\mathrm{n}=1, \mathrm{MW}=0,0120), 1980(\mathrm{n}=8$, MW=0,0199), 1986 ( $n=12, M W=0,0044)$, $1995(\mathrm{n}=3, \mathrm{MW}=0,0003)$ und $2005(\mathrm{n}=3$, $\mathrm{MW}=0,0087$ ). Die Expositionsschätzungen wurden auf diese 5 Mittelwerte der Konzentration pro Kalenderjahr gegrün- 

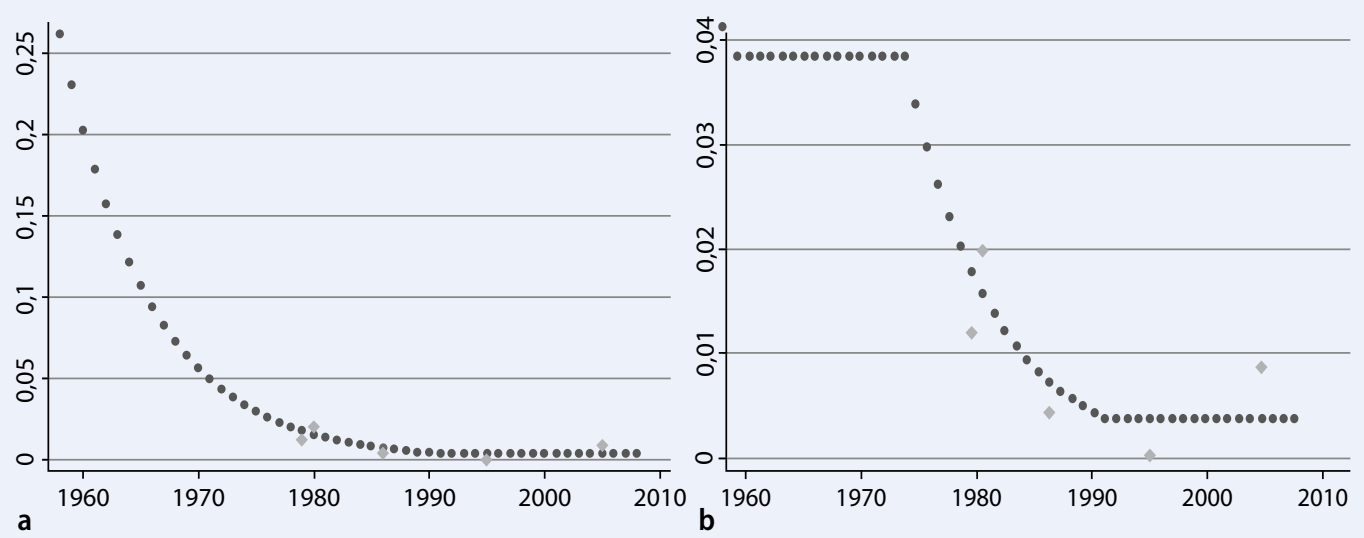

Abb. $2 \Delta$ Rückwärtsextrapolation der Belastungshöhen gegenüber Cyanurchlorid. a Verlauf der Expositionsschätzung mit einem unrealistisch hohen rückwärtigen Anstieg bis zum Beginn der Produktion auf Basis der log-linearen Regression mit konstantem Endniveau (Kreise) zu 5 Jahresmittelwerten der Messungen (Rauten). b Konstante Deckelung des Anstiegs aus der log-linearen Regression mit dem Geruchsschwellenwert auf Basis des Expertenwissens. Konstantes Endniveau ab 1992. Mittelwerte der Messungen als Rauten dargestellt. Durchschnittskonzentration in $\mathrm{mg} / \mathrm{m}^{3}$ zur Leitexpositionsschätzung ( $\mathrm{mw}_{-}$ nwg2_umr) am Beispiel des Anlagenfahrers (Jobkategorie: Produktionsmitarbeiter) in der Messwarte des Werks Wesseling

det. Mit den Messungen aus 1995 und 2005 wurde ein Endniveau ab 1991 geschätzt $(\mathrm{MW}=0,0045)$. Davon rückwärts ausgehend wurde durch die Jahresmittelwerte vor 1995 eine log-lineare Regression gelegt. $\bullet$ Abb. 2a zeigt die Jahresmittelwerte der Messungen, das Endniveau und den Verlauf der mit einem log-linearen Regressionsmodell in die Vergangenheit extrapolierten Messdaten. Diese Auswertung der Messdaten belegt beispielhaft den auch von den Experten grundsätzlich berichteten ausgeprägten Rückgang der Cyanurchloridkonzentrationen mit der Zeit. Der Fit der Ausgleichskurve erscheint hinreichend gut, um diese Funktion zur Imputation fehlender Daten zu verwenden. Die Extrapolation dieser loglinearen Kurve führte aber zu einem unrealistisch hohen rückwärtigen Anstieg der Belastung bis zum Beginn der Produktion. Daher wurde auf Basis des Expertenwissens eine konstante Deckelung des rückwärtigen Anstiegs mit dem Geruchsschwellenwert bei $0,0385 \mathrm{mg} / \mathrm{m}^{3}$ vorgenommen. Die Beschreibung der historischen Situation durch die Experten belegte, dass auch in frühen Zeiten an diesem Arbeitsplatz die Geruchsschwelle nicht überschritten wurde. Das Resultat war ein plausiblerer Verlauf der Belastungshöhe gegenüber Cyanurchlorid über den gesamten Produktionszeitraum (- Abb. 2b). Alle Auswertungen stützen sich auf kumulierte Expositionsmaße, die bis hin zur Erhebung der medizinischen
Endpunkte integriert werden, sodass Knickpunkte oder unstetige Entwicklungen in der Konzentrationsschätzung über der Zeit tolerabel sind.

Neben diesem log-linearen Modellansatz wurden in anderen Messort/SEGKombinationen nach Expertenurteil und Datenlage Stufenmodelle, Modelle mit linearer Extrapolation oder Modelle mit Imputation von Mittelwerten anderer Werke für die Rückwärtsextrapolation gewählt (vollständige Dokumentation dieser Vorgehensweise und der zur Verankerung benutzten Messdatenanalyse beim Verfasser).

\section{Aufbereitung der personenbezogenen Daten}

Aus dem Teildatenkörper HSA wurden sowohl die Arbeitsgeschichten aus der Personalabteilung als auch die Daten aus den arbeitsmedizinischen Untersuchungen aufbereitet. Die erfassten individuellen Tätigkeiten wurden entsprechend der harmonisierten Jobkategorien des Teildatenkörpers JEM den vier definierten Jobkategorien zeitabhängig zugewiesen. Somit wurde für jede Person ein Tätigkeitsverlauf, beschrieben in den 4 Jobkategorien, erstellt. Die angegebenen Vorbelastungen wurden nach ISIC [26] verdichtet und in Vorbelastungsdauern umgerechnet.

Die aus den arbeitsmedizinischen Untersuchungen gewonnenen Daten wurden Plausibilitätsprüfungen unterzogen, um z. B. Größe und Gewicht der Stu- dienteilnehmer auf Ausreißer zu prüfen: Abweichungen wurden zur Überprüfung durch den Eingebenden gekennzeichnet und nach Überprüfung der Originaldokumente ggf. korrigiert. Bei der Überprüfung der Rauchangaben wurden die Zeitangaben zueinander in Beziehung gesetzt und so auf Plausibilität geprüft (z. B. Alter des Studienteilnehmers zu Rauchdauer, Rauchbeginn zu Rauchende etc.).

Für eine einheitliche Darstellung des Rauchverhaltens als Voraussetzung für eine vergleichende Auswertung der Rauchangaben wurden Pack years berechnet. Dabei werden die Rauchmengen einer Person berechnet als Zigarettenpackungen pro Tag, multipliziert mit der Zahl der Raucherjahre (bei einer Annahme von 20 Zigaretten/Packung). Die Anzahl der Pack years wird bis zum letzten Datensatz eines Studienteilnehmers kumuliert. Bei fehlenden Angaben für Rauchmenge bzw. Rauchdauer wurde soweit möglich - eine Vervollständigung der Daten auf Basis der vorhandenen Angaben durch Imputation der aus allen Rauchangaben errechneten Mittelwerte durchgeführt.

Aus den pro Person und Untersuchungsdatum ermittelten Lungenfunktionsparametern FVC und VC wurde zur Vorbereitung der weiteren Analysen der Maximalwert $\mathrm{VC}_{\max }=\max (\mathrm{VC}, \mathrm{FVC}$ ) ermittelt. Die Lungenfunktionsparameter $\mathrm{VC}_{\max }, \mathrm{FEV}_{1}$ und der Tiffeneau-Wert $\mathrm{FEV}_{1} \% \mathrm{FVC}$ wurden zudem in Prozent 
Tab. 4 Cyanurchloridbelastung für jedes Werk und gesamt (Leitexpositionsschätzung)

\begin{tabular}{|c|c|c|c|c|}
\hline $\begin{array}{l}\text { Betriebsstand- } \\
\text { orte }\end{array}$ & Antwerpen & Münchsmünster & Wesseling & Gesamt \\
\hline $\begin{array}{l}\text { Anzahl Studien- } \\
\text { teilnehmer }\end{array}$ & 144 & 120 & 130 & 394 \\
\hline \multicolumn{5}{|c|}{ Gesamt-Durchschnittskonzentration $\left(\mathrm{mg} / \mathrm{m}^{3}\right)$} \\
\hline $\begin{array}{l}\text { Arithm. } \\
\text { Mittelwert }\end{array}$ & 0,016 & 0,028 & 0,014 & 0,019 \\
\hline Maximum & 0,028 & 0,044 & 0,030 & 0,044 \\
\hline \multicolumn{5}{|c|}{ Gesamt-Expositionsdauer (Jahre) } \\
\hline $\begin{array}{l}\text { Arithm. } \\
\text { Mittelwert }\end{array}$ & 12,98 & 8,11 & 12,23 & 11,25 \\
\hline Maximum & 33,92 & 31,77 & 32,00 & 33,92 \\
\hline \multicolumn{5}{|c|}{ Kumulierte Gesamt-Exposition (mg/m³-Jahre) } \\
\hline $\begin{array}{l}\text { Arithm. } \\
\text { Mittelwert }\end{array}$ & 0,216 & 0,244 & 0,192 & 0,217 \\
\hline Maximum & 0,674 & 0,941 & 0,894 & 0,941 \\
\hline
\end{tabular}

Tab. 5 Cyanurchloridbelastung (Leitexpositionsschätzung) für das Werk Wesseling ab 2000 und für alle Untersuchungsjahre der Studie (in Klammern)

\begin{tabular}{|lllll}
\hline Jobkategorien & Austragsmitarbeiter & $\begin{array}{l}\text { Produktions- } \\
\text { mitarbeiter }\end{array}$ & Labormitarbeiter & Gesamt \\
\hline Gesamt-Durchschnittskonzentration $\left(\mathrm{mg} / \mathrm{m}^{3}\right)$ & & \\
\hline Arithm. Mittelwert & 0,010 & 0,010 & 0,017 & 0,010 \\
& $(0,014)$ & $(0,014)$ & $(0,023)$ & $(0,014)$ \\
\hline Maximum & 0,010 & 0,046 & 0,018 & 0,046 \\
& $(0,023)$ & $(0,030)$ & $(0,029)$ & $(0,030)$ \\
\hline Gesamt-Expositionsdauer (Jahre) & & & 4,91 \\
\hline Arithm. Mittelwert & 7,44 & 4,77 & 5,23 & $(12,23)$ \\
\hline & $(8,23)$ & $(11,79)$ & $(19,83)$ & 8,00 \\
\hline Maximum & 8,00 & 6,25 & 7,84 & $(32,00)$ \\
\hline & $(18,72)$ & $(27,44)$ & $(32,00)$ & 0,046 \\
\hline Kumulierte Gesamt-Exposition $\left(\mathrm{mg} / \mathrm{m}^{3}\right.$-Jahre) $)$ & & $(0,192)$ \\
\hline Arithm. Mittelwert & 0,073 & 0,042 & 0,119 \\
& $(0,111)$ & $(0,178)$ & $(0,472)$ & $(0,894)$ \\
\hline Maximum & 0,078 & 0,052 & 0,119 & \\
\hline & $(0,338)$ & $(0,513)$ & $(0,894)$ & 0,046 \\
\hline Kumulierte Gesamt-Exposition $\left(\mathrm{mg} / \mathrm{m}^{3}\right.$-Jahre) $)$ & & $(0,192)$ \\
\hline Arithm. Mittelwert & 0,073 & 0,042 & 0,119 \\
& $(0,111)$ & $(0,178)$ & 0,086 & $(0,894)$ \\
\hline Maximum & 0,078 & 0,052 & $(0,472)$ & \\
& $(0,338)$ & $(0,513)$ & 0,119 & $(0,894)$ \\
\hline
\end{tabular}

vom EU-Sollwert berechnet, der von Geschlecht, Alter und Körpergröße abhängt [47]. Um eine Verzerrung der Ergebnisse durch verschiedene Messgeräte bewerten zu können, wurden die in dieser Studie verwendeten Lungenfunktionsmessgeräte zeitabhängig in die 4 Messgerätegruppen Glockenspirometer, Vitalograph, CustoVit und Bodyplethysmograph, unterteilt.

\section{Vorbereitung der Zusammenhangsanalyse}

Die beiden Teildatenkörper JEM und HSA wurden über die Jobkategorie zu einem finalen Auswertungsdatensatz auf Monatsebene zusammengeführt. Über die entwickelte Job-Exposure-Matrix konnten allen Studienteilnehmern SEG und Messort und damit die entsprechende Cyanurchloridbelastung in Form von Konzentration und Dauer sowie die Rotationsdauern für Tätigkeiten außerhalb der Cyanurchloridanlage zugewiesen werden. Als nächstes wurde ein finaler Auswertungsdatensatz auf Jahresebene gebildet, der für alle Studienteilnehmer die durchschnittliche Cyanurchloridkonzentration (1), die pro Jahr kumulierte Cyanurchlo- ridexpositionsdauer (2) und die kumulierte Cyanurchloridexposition [Produkt aus (1) und (2)] enthielt. Die Berechnungen erfolgten jeweils bis einschließlich des Kalenderjahres der letzten medizinischen Untersuchung des Studienteilnehmers.

Da in Münchsmünster eine Kollinearität zwischen $\mathrm{CaCl}_{2}$ und $\mathrm{NDC}$ bzw. $\mathrm{CaCl}_{2}$ und $\mathrm{ACH}$ vorlag, wurden die Kovariablen reduziert. NDC und $\mathrm{ACH}$ wurden nicht weiter betrachtet, denn eine Berücksichtigung des potenziellen Confounders $\mathrm{CaCl}_{2}$ im Regressionsmodell adjustiert aufgrund der Kollinearität gleichzeitig für ein Confounding durch NDC und ACH. In den Werken Antwerpen und Wesseling fand nur eine Rotation mit der $\mathrm{HCl}_{(\mathrm{aq})}$-Anlage statt; diese Variable wurde ebenfalls zur Confounderadjustierung mitgeführt. Um den Umfang der Rotationen besser in den Auswertungen berücksichtigen zu können, werden diese Koexpositionen nicht als Indikatorvariablen aufgebaut, sondern es wurden die kumulierten Dauern der Rotationen ermittelt. In den Regressionsmodellen werden somit die Rotationsdauern für die Chemikalien $\mathrm{CaCl}_{2}, \mathrm{HCN}$ und $\mathrm{HCl}_{(\mathrm{aq})}$ und die $\mathrm{HCl}_{(\mathrm{aq})}$-Koexposition als Kovariablen zur Confounderadjustierung mitgeführt.

\section{Ergebnisse}

\section{Kohorte}

Der Gesamtumfang der Studiengruppe betrug zunächst 427 Studienteilnehmer, für die über die HSA-Software Arbeitsgeschichten und medizinische Daten erfasst wurden. Nachträglich ausgeschlossen wurden Personen ohne medizinische Akte $(n=14)$, ohne Cyanurchloridbelastung $(\mathrm{n}=5)$ und Personen, die insgesamt weniger als ein Jahr in Cyanurchloridbetrieben tätig waren $(\mathrm{n}=14)$. Das finale Studienkollektiv umfasste 394 Studienteilnehmer, die sich weitgehend gleichmäßig (120/130/144) auf die Werke verteilten. Insgesamt wurden 10.661 Datensätze erhoben, davon 2989 Datensätze zu medizinischen Angaben und 7672 Datensätze zu den Tätigkeiten der Studienteilnehmer.

Die Studienteilnehmer waren überwiegend Deutsche $(\mathrm{n}=234)$ und Westeuropäer $(\mathrm{n}=138)$. Die restlichen Studienteilnehmer $(n=22)$ wurden aufgrund der gerin- 


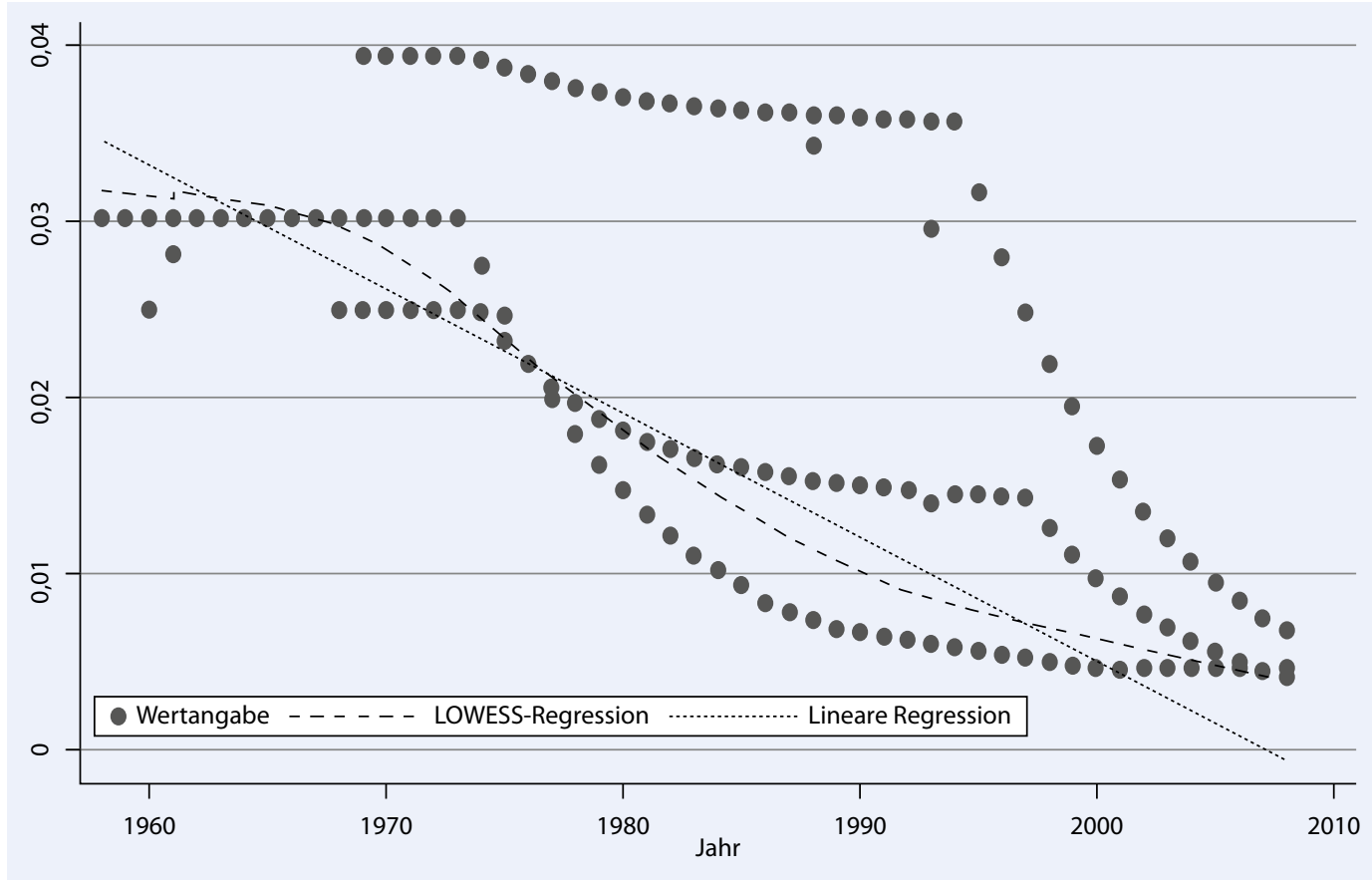

Abb. $3<$ Mittlere Cyanurchloridkonzentration pro Expositionsjahr in $\mathrm{mg} / \mathrm{m}^{3}$ für das Werk Wesseling mit linearer Regression und LOWESS-Regression über der Kalenderzeit (130 exponierte Personen, 2212 Personen-Jahre, Mittelwert $=0,015 \mathrm{mg} / \mathrm{m}^{3}$, $\mathrm{SD}=0,010 \mathrm{mg} / \mathrm{m}^{3}$, $\min .=0,006 \mathrm{mg} / \mathrm{m}^{3}$, $\max .=0,032 \mathrm{mg} / \mathrm{m}^{3}$ )

gen Anzahl zu einer Gruppe zusammengefasst. Von den 394 Studienteilnehmern waren 221 zum Zeitpunkt der Studie noch aktiv in einem Cyanurchloridbetrieb tätig, während 173 vor Beginn der Studie den Cyanurchloridbetrieb verlassen hatten. Bei der letzten medizinischen Untersuchung lag das durchschnittliche Alter der Studienteilnehmer bei 47,5 Jahren ( $\mathrm{SD}=10,3$ Jahre). Der jüngste Studienteilnehmer war 21 und der älteste 63 Jahre alt: 9 Männer waren jünger als 25 Jahre.

\section{Exposition gegenüber Cyanurchlorid}

Personenbezogene Cyanurchloridkonzentrationen wurden in 24 Versionen aus der eingeschränkten, im Wesentlichen aktuelleren Messdatenbasis geschätzt und durch Extrapolation aufgrund von Expertenschätzungen in die Vergangenheit verlängert. Im Folgenden wird zu einer typischen Schätzung im Sinne einer Leitexpositionsschätzung berichtet: Anwendung a) des Mittelwertes als Stützstatistik, b) der Umrechnungsfaktoren zwischen den Messverfahren und c) der Imputation der halben Nachweisgrenze bei Messwerten unterhalb der Nachweisgrenze (kurz: mw_nwg2_umr, • Tab.3).

Die personenbezogene Durchschnittskonzentration der Cyanurchloridexposi- tion betrug über alle Jahre im Mittel $0,019 \mathrm{mg} / \mathrm{m}^{3}$ ( $\left.\min .=0,003, \max .=0,044\right)$ bei einer Expositionsdauer von im Mittel 11,3 Jahren $(\min .=0,58, \max .=34)$. Die kumulierte Gesamtexposition lag im Mittel bei $0,217 \mathrm{mg} / \mathrm{m}^{3}$-Jahre (min. $=0,005$, max.=0,941). Der Mittelwert der Cyanurchloridkonzentration pro Expositionsjahr betrug $0,020 \mathrm{mg} / \mathrm{m}^{3}$ ( $\mathrm{min} .=0,003$, max.=0,051). - Tab. 4 gibt einen Überblick über die Expositionsvariablen im Querschnitt zum Zeitpunkt der letzten arbeitsmedizinischen Untersuchung für die einzelnen Werke und insgesamt.

Seit 2008 wird nur im Werk Wesseling weiterhin Cyanurchlorid produziert. Um eine realistische Einschätzung der aktuellen Cyanurchloridexposition vornehmen zu können, wurden in $\bullet$ Tab. 5 die dort ab 2000 gemessenen Expositionen aufgeteilt nach Jobkategorien dargestellt. In Klammern sind zusätzlich Expositionsschätzungen über alle Untersuchungsjahre, die insgesamt in die Studie für Wesseling eingegangen sind, angegeben. Ein Vergleich zeigt eine tendenziell niedrigere Belastung ab 2000 .

- Abb. 3 stellt den Verlauf der geschätzten Cyanurchloridkonzentration pro Kalenderjahr für das Werk Wesseling über alle Untersuchungsjahre dar. In der Abbildung trennen sich die Verläufe für unterschiedliche Tätigkeitsgruppen.
Dargestellt sind zur Beschreibung der mittleren Konzentrationsentwicklung im Kollektiv auch eine Gerade (lineare Regression) und eine nicht-parametrisch glättende Verlaufskurve (LOWESSRegression, „locally weighted scatterplot smoothing "), die sich allerdings nur unbedeutend voneinander unterscheiden.

\section{Vorbelastungen}

Die Vorbelastungen der Studienteilnehmer wurden vor Beginn einer Beschäftigung in einem Cyanurchloridbetrieb, die auf eine Belastung der Lunge als Zielorgan hinweisen könnten, erfasst und mittels ISIC verdichtet [26].

Während in Antwerpen keine dokumentierten Vorbelastungen außerhalb des Chemiebereichs vorlagen, waren in Münchsmünster und Wesseling Informationen $\mathrm{zu}$ weiteren Vorbelastungen vorhanden, wobei hier der größte Anteil aller Vorbelastungen auf den Chemiebereich entfiel. Insgesamt 233 von 394 Personen gaben an, vor der Aufnahme ihrer Tätigkeit in dem Cyanurchloridbetrieb in einem anderen Bereich der Chemieindustrie gearbeitet zu haben. Auch wenn berücksichtigt wird, dass für Studienteilnehmer aus Antwerpen ausschließlich Vorbelastungsangaben aus dem Bereich Chemie vorlagen, trat die Angabe Chemie 

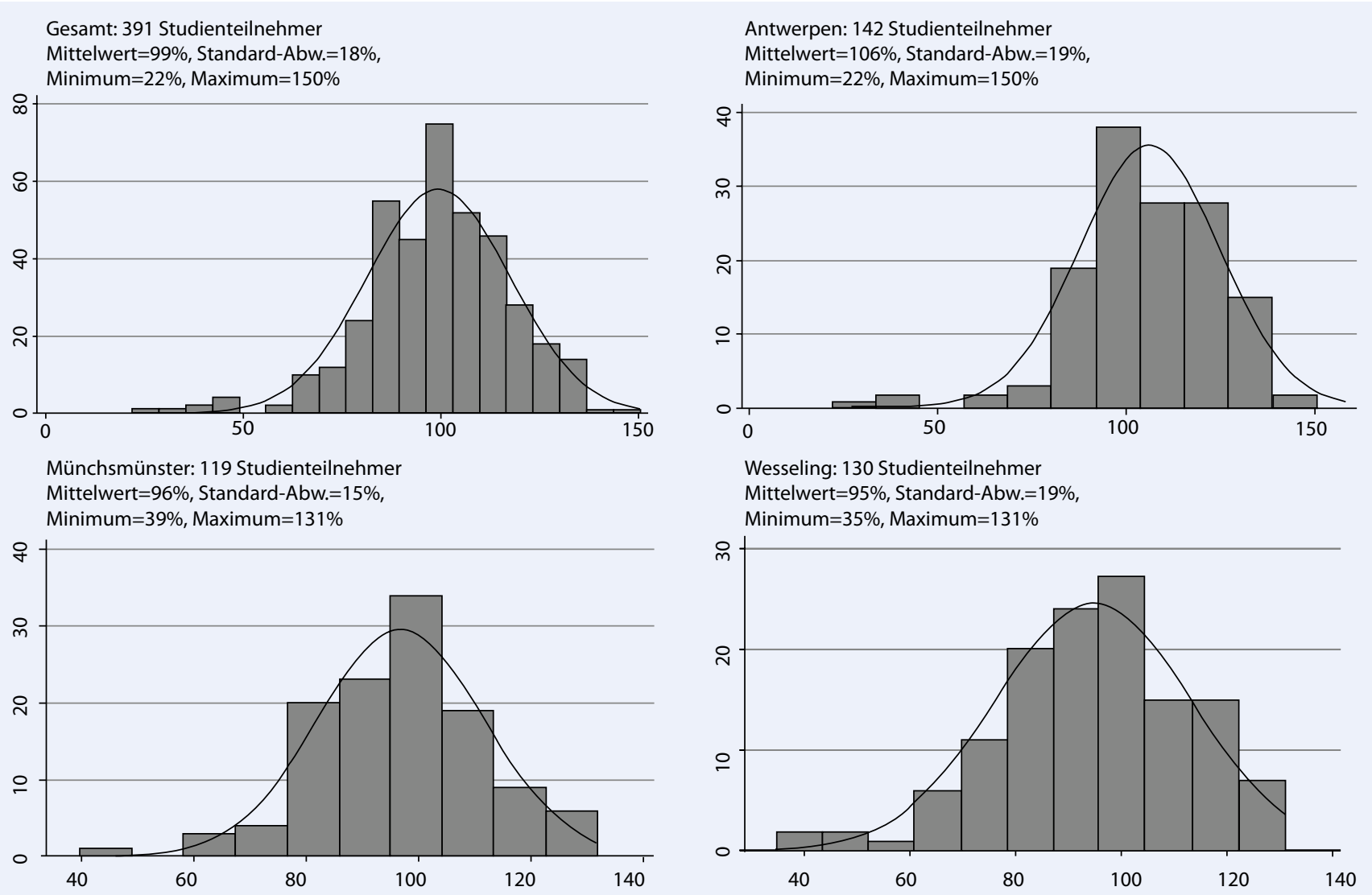

Abb. $4 \Delta$ Verteilung des Lungenfunktionsparameters $\mathrm{FEV}_{1}$ bezogen auf Quanjer-Sollwerte in \% bei Studienende, getrennt nach Werken und gesamt

mehr als doppelt so häufig auf wie alle weiteren angegebenen Vorbelastungen.

\section{Rauchverhalten}

Angaben zum Rauchverhalten lagen für insgesamt 387 Studienteilnehmer vor (Antwerpen 144, Münchsmünster 113, Wesseling 130). Von den 397 Studienteilnehmern waren bei mindestens einer Untersuchung 160 aktive Raucher (Antwerpen 44, Münchsmünster 52, Wesseling 64), 78 Ex-Raucher (Antwerpen 16, Münchsmünster 30, Wesseling 32) und 149 waren Nie-Raucher (Antwerpen 84, Münchsmünster 31, Wesseling 34). Der Mittelwert für die zum Zeitpunkt der letzten Lungenfunktionsuntersuchung aufgelaufenen Pack years lag für die Gruppe der aktiven Raucher bei 18,94 (SD=14,93) und für die Gruppe der Ex-Raucher bei 19,41 $(\mathrm{SD}=18,37)$. In Antwerpen gab es somit einen im Vergleich zu den anderen Standorten hohen Anteil an Nie-Rauchern (58\%). Dieses in Abhängigkeit von den
Werken deutlich unterschiedliche Rauchverhalten zeigte sich auch in den ähnlich variierenden Pack years-Angaben, d. h. der Ausprägung des Rauchens unter den Rauchern. Für aktive Raucher (Ex-Raucher) ergaben sich folgende mittlere Werte: Antwerpen 15,2 (13,9), Münchsmünster 19,5 (17,4), Wesseling 21,2 $(24,0)$.

\section{Respiratorische Symptome und Erkrankungen}

157 Studienteilnehmer haben über den Fragebogen zu Atemwegserkrankungen und Rauchgewohnheiten Angaben zu Atemnot, Asthma, chronischer Bronchitis bzw. zu COPD abgegeben. Atemnot ( $\mathrm{n}=5$ Fälle) und die COPD-Stufen Ib (n=1), IIb $(\mathrm{n}=0)$, IIc $(\mathrm{n}=0)$, IIIa $(\mathrm{n}=8)$, IIIb $(\mathrm{n}=0)$, IVa $(n=1)$, IVb $(n=0)$ wurden relativ selten oder gar nicht genannt. Lediglich bei Asthma ( $\mathrm{n}=33)$, chronischer Bronchitis $(\mathrm{n}=14)$ und den COPD-Stufen Ia $(\mathrm{n}=38)$ und IIa $(n=26)$ lagen ausreichende Fallzahlen vor, die weitergehende Analy- sen im Querschnitt indizieren. Während Angaben $\mathrm{zu}$ Asthma und chronischer Bronchitis nur im Querschnitt verfügbar waren, konnten die aus den Lungenfunktionsmessungen ermittelten COPD-Stufen Ia und IIa sowohl im Querschnitt als auch im Längsschnitt dargestellt werden. Eine positive Einstufung in die COPDStufen Ia und IIa erfolgte für 391 Personen im Längsschnitt. Die COPD-Stufe Ia lag bei 74 Personen vor. Von der COPDStufe IIa waren 53 Personen betroffen.

\section{Sensibilisierungsparameter}

Für 130 Studienteilnehmer lagen immunserologische Untersuchungen zur Bestimmung einer allgemeinen Sensibilisierung (Gesamt-IgE) und einer Sensibilisierung gegenüber Cyanurchlorid (spezifischer IgE) vor. Bei den insgesamt 130 Werten für den spezifischen IgE gab es 22 Werte $(16,9 \%)$ oberhalb der Nachweisgrenze von $0,1 \mathrm{kU} / \mathrm{l}$. Alle Werte unterhalb der NWG wurden für weitere Analysen auf den hal- 


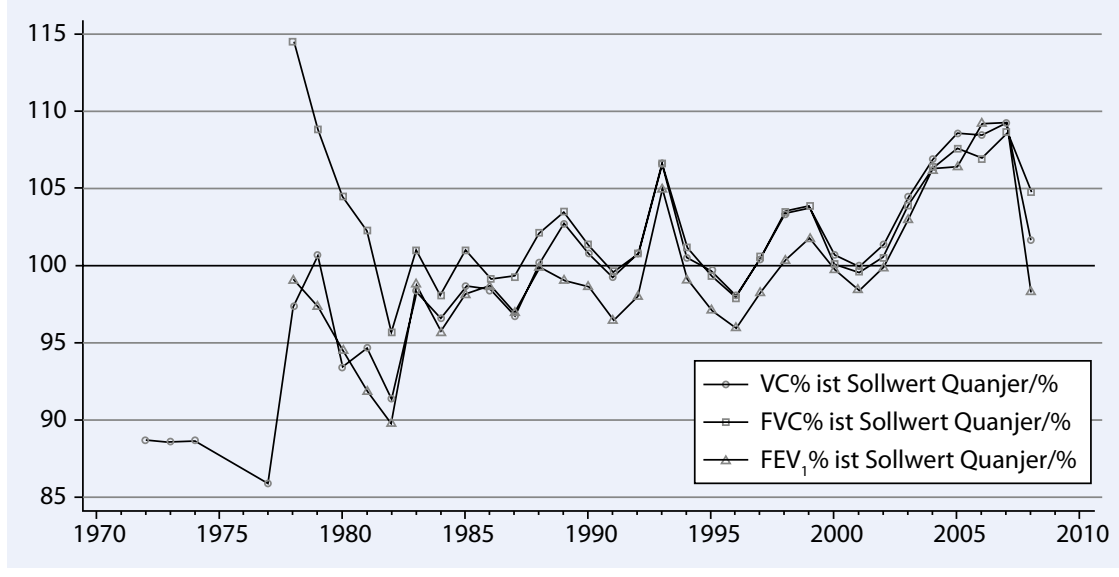

Abb. $5 \Delta$ Lungenfunktionsmesswerte bezogen auf Quanjer-Sollwerte in \% über der Kalenderzeit für alle Werke. Jahresmittelwerte der VC (390 Studienteilnehmer; 3031 Untersuchungen), FVC (393; 2956) und FEV $1(391 ; 2953)$

ben Wert der NWG gesetzt. Bei den Gesamt-IgE-Werten dagegen lagen alle 130 gemessenen Werte (100\%) oberhalb der NWG von $0,2 \mathrm{kU} / \mathrm{l}$.

Es erfolgte eine Kategorisierung der Sensibilisierungsparameter mit Bezug auf den entsprechenden Referenzwert, der für den spezifischen IgE-Wert bei $0,35 \mathrm{kU} / \mathrm{l}$ und für den IgE-Wert bei $300 \mathrm{kU} / \mathrm{l}$ liegt. Bei insgesamt 15 von 130 Werten (11,5\%) lag der spezifische IgE-Wert oberhalb des Referenzwerts. Bei dem Gesamt-IgE-Wert lagen 11 von 130 (8,5\%) oberhalb des Referenzwerts. Der Anteil an auffälligen Werten des spezifischen IgE und des GesamtIgE lag in Münchmünster mit 17,1\% (12 von 70 ) bzw. 14,3\% (10 von 70) deutlich höher als in Antwerpen (4,3 bzw. 0\%, von 23) und Wesseling (5,4 bzw. 2,7\%, von 37).

Der spezifische IgE-Wert betrug im Mittel über alle Werke 1,13 kU/l (SD=4,57, $\min .=0,05$, max. $=34$ ). In Antwerpen betrug z. B. der Mittelwert $0,40 \mathrm{kU} / \mathrm{l}$, in Münchsmünster dagegen 1,88 kU/l und in Wesseling 0,19 kU/l. Der Gesamt-IgEWert betrug im Mittel über alle Werke $123 \mathrm{kU} / \mathrm{l}(\mathrm{SD}=230, \min .=2$, $\max .=2009)$. In Antwerpen betrug z. B. der Mittelwert $73 \mathrm{kU} / \mathrm{l}$, in Münchsmünster dagegen $161 \mathrm{kU} / \mathrm{l}$ und in Wesseling $82 \mathrm{kU} / \mathrm{l}$. In den stetigen Auswertungen wiederholt sich somit der Befund der höheren Werte in Münchsmünster.

\section{Lungenfunktion}

Für alle Studienteilnehmer lagen Angaben zu Lungenfunktionsparametern vor. Für 393 von 394 Studienteilnehmern waren Angaben zur FVC, für 1 Studienteilnehmer waren nur Angaben zur VC vorhanden. Im Durchschnitt standen für jeden Studienteilnehmer 7,39 FVC-Untersuchungen (min. $=1$; max. $=19$ ) zur Verfügung. Der FVC\%Sollwert(Quanjer) betrug bei der ersten Lungenfunktionsuntersuchung jedes Studienteilnehmers in der Studiengruppe im Mittel 102,7\% $(\mathrm{s}=14,2 \%)$. Der Mittelwert der FVC\%Sollwert(Quanjer) bei der letzten Untersuchung jedes Studienteilnehmers im Datensatz lag bei $103,4 \%$. ( $S D=15,4 \%)$. Die Werte der FVC\%Sollwert(Quanjer) bei der letzten Untersuchung stellten sich als annähernd normal verteilt dar. Die zusätzlich verwendeten Referenzwerte nach GLI ergaben einen Mittelwert der FVC\%Sollwert(GLI) bei der ersten Untersuchung von $95,7 \%$ ( $\mathrm{SD}=12,9 \%$ ) bzw. bei der letzen Untersuchung von $95,8 \%(S D=14,1 \%)$. Für 391 Studienteilnehmer waren Angaben zur FEV ${ }_{1}$ vorhanden. Die durchschnittliche Anzahl verfügbarer $\mathrm{FEV}_{1}$-Messungen belief sich auf $n=7,42$ Werte pro Person $(\min .=1 ; \max .=19)$. Der Mittelwert der FEV $_{1} \%$ Sollwert(Quanjer) zur ersten Untersuchung je Studienteilnehmer betrug 99,1\% (SD=15,4\%). Der Mittelwert der $\mathrm{FEV}_{1} \%$ Sollwert(Quanjer) bei der letzten Untersuchung jedes Studienteilnehmers lag bei $99,4 \%(S D=18,3 \%)$. Die GLI-Referenzwerte $\mathrm{FEV}_{1} \%$ Sollwert(GLI) betrugen im Mittel bei der ersten Untersuchung $94,4 \%$ ( $\mathrm{SD}=14,4 \%$ ) und bei der letzten Untersuchung 93,9\% (SD=17,2\%). Die Verteilung der Messwerte bei der letzten
Untersuchung ist, wie bei der FVC, ebenfalls als annähernd normal anzusehen.

Angaben zur $\mathrm{FEV}_{1} \% \mathrm{FVC}$ waren für 391 Studienteilnehmer vorhanden. Die Angaben zur $\mathrm{FEV}_{1} \% \mathrm{FVC}$ lagen nur für Untersuchungen vor, bei denen beide Parameter mit dem gleichen Lungenfunktionsmesssystem in der gleichen Untersuchung bestimmt wurden. Es standen im Mittel n=7,42 Angaben (min. $=1$; max. $=19$ ) je Studienteilnehmer für die Auswertung zur Verfügung. Bei der ersten Untersuchung des Studienteilnehmers betrug der Mittelwert für die $\mathrm{FEV}_{1} \% \mathrm{FVC} \%$ Sollwert(Quanjer) 99,2\% (SD=10,4\%). Die Verteilung der Werte zur ersten Untersuchung ist ähnlich wie die für die FVC und $\mathrm{FEV}_{1}$. Der Mittelwert der $\mathrm{FEV}_{1} \% \mathrm{FVC} \%$ Sollwert(Quanjer) bei der letzten Untersuchung je Studienteilnehmer errechnete sich zu 98,8\% (SD=12,2\%) und war damit minimal geringer als bei der ersten Untersuchung. Die GLI-Referenzwerte $\mathrm{FEV}_{1} \% \mathrm{FVC} \%$ Sollwert(GLI) betrugen im Mittel bei der ersten Untersuchung 98,2\% ( $\mathrm{SD}=9,9 \%$ ) und bei der letzten Untersuchung $97,6 \%$ ( $\mathrm{SD}=11,9 \%)$.

- Abb. 4 zeigt die Verteilung der $\mathrm{FEV}_{1}$-Werte bezogen auf die EU-Sollwerte nach Quanjer et al. [46] für das gesamte Kollektiv und für die 3 Teilkollektive Antwerpen, Münchsmünster und Wesseling. Dargestellt sind die Werte am Ende des Studienzeitraums, also nach individuell höchster kumulierter Exposition gegenüber Cyanurchlorid.

Die Entwicklung der Lungenfunktionsparameter über die Zeit ist in - Abb. 5 dargestellt. Die Abbildung ergibt keine fallenden Werte.

\section{Diskussion und Zusammenfassung}

Für eine Studiengruppe von 394 Männern, die zwischen 1958 und 2007 in cyanurchloridproduzierenden Betrieben tätig waren, wurden medizinische Daten, Tätigkeiten und Expositionen erfasst und verarbeitet. Es handelt sich um eine retrospektive Kohortenstudie, die - nach Möglichkeit - alle aktiven und ehemaligen Mitarbeiter der drei Cyanurchloridbetriebe Antwerpen, Münchsmünster und Wesseling umfasst. Das Studienkollektiv wurde beschränkt auf männliche Mitarbeiter, 
die mindestens ein Jahr im Cyanurchloridbetrieb tätig waren. Im Rahmen einer Längsschnittuntersuchung wurden medizinische Daten und Tätigkeitsprofile der Studienteilnehmer sowie die Expositionsdaten von Produktionsbeginn bis zum Studienende 2007 erfasst.

Die Datenerhebung fand unter Einhaltung der Datenschutzkriterien zur Erfassung personenbezogener Daten über eine unter diesem Aspekt entwickelte Software statt. Zur Erfassung der medizinischen Daten wurden die im arbeitsmedizinischen Dienst des jeweiligen Betriebs vorliegenden medizinischen Akten für alle aktiven und historischen Studienteilnehmer herangezogen. Erfasst wurden alle arbeitsmedizinischen Untersuchungen mit Lungenfunktionsprüfung. Dies eröffnet die Möglichkeit, über einen langen Zeitraum die Auswirkungen der Cyanurchloridbelastung auf die Lungenfunktion von Mitarbeitern in Cyanurchloridbetrieben zu untersuchen. Neben den Lungenfunktionsparametern wurden Alter, Herkunftsland, biometrische Daten und Rauchgewohnheiten dokumentiert. Für die aktiven Studienteilnehmer fand aufgrund der Studie eine zusätzliche medizinische Untersuchung statt, in der neben einer weiteren Lungenfunktionsuntersuchung auch eine Blutuntersuchung zur Bestimmung von Sensibilisierungsparametern durchgeführt wurde. Zur Überprüfung von Atemwegserkrankungen und Rauchgewohnheiten wurde von den aktiven Studienteilnehmern ein detaillierter Fragenbogen ausgefüllt, der ebenfalls elektronisch erfasst wurde.

Das durchschnittliche Alter der Studienteilnehmer lag bei 47,5 Jahren ( $\mathrm{min} .=21$, max. $=63, S D=10,3)$. Es gab 160 aktive Raucher, 78 Ex-Raucher und 149 NieRaucher.

Für die Modellierung der Messdaten wurden die vorliegenden Cyanurchloridkonzentrationsdaten aus den vorhandenen Messprotokollen unter Berücksichtigung des Tragens und der Wirksamkeit von Schutzmasken ausgewertet. Bei fehlenden Angaben wurden Expositionshöhe und Expositionsverlauf über Expertenschätzungen vervollständigt. Für diese Studie wurde eine JEM entwickelt, über die die entsprechende Cyanurchloridbelastung den einzelnen Tätigkeiten der Stu- dienteilnehmer zugewiesen werden konnte.

Die vorhandenen Cyanurchloridmessdaten der einzelnen Werke waren nicht ausreichend für eine direkte Messdatenmodellierung über den gesamten Produktionszeitraum der Werke. Um die innewohnenden Unsicherheiten abzubilden, wurden verschiedene Expositionsszenarien ermittelt. Die Messwerte wurden zunächst auf eine Messmethode und eine Messart normiert, durch Expertenschätzungen vervollständigt, bei Bedarf rückwärtsextrapoliert und verschiedenen Annahmen unterworfen. Insgesamt ergaben sich damit für die multiplen Expositionsschätzungen $24 \mathrm{Va}$ rianten zur Ermittlung der Cyanurchloridexposition. Diese multiple Expositionsschätzung erlaubt eine Sensitivitätsanalyse innerhalb der epidemiologischen Auswertung durchzuführen, sodass möglicherweise robuste Schlussfolgerungen aus der Messdatenlage gezogen werden können.

Zur Vorbereitung der Zusammenhangsanalyse wurde jedem Studienteilnehmer über eine für diese Studie entwickelte JEM die seiner Tätigkeit entsprechende Cyanurchloridbelastung und seine medizinischen Daten aus den arbeitsmedizinischen Untersuchungen zugewiesen. Durch Rotation in den Betrieben war ein großer Teil der Studienteilnehmer nur in begrenzten Zeitabschnitten einer Cyanurchloridbelastung ausgesetzt. Dies wurde bei Ermittlung der Langzeitexpositionen individuell berücksichtigt. Zu den Rotationszeiten wurden Expositionsdaten zu solchen Chemikalien als Kovariablen erhoben, die eine Reizwirkung auf die Atemwege ausüben oder zu Veränderungen der Lungenfunktion führen könnten. Vorexpositionen aus anderen Beschäftigungsverhältnissen in der chemischen Industrie wurden ebenfalls dokumentiert.

Für eine ausgewählte Variante (Leitexpositionsschätzung) wurde die Expositionssituation ermittelt (Mittelwerte als Stützstatistik, Anwendung der Umrechnungsfaktoren zwischen den Messverfahren und Imputation der halben Nachweisgrenze bei Messwerten unterhalb der Nachweisgrenze). Die personenbezogene Durchschnittskonzentration der Cyanurchloridexposition betrug in dieser Metrik über alle Jahre im Mittel $0,019 \mathrm{mg} / \mathrm{m}^{3}$ ( $\mathrm{min} .=0,003$, $\left.\max .=0,044\right)$ bei einer Expositionsdauer von im Mittel 11,3 Jahren (min.=0,58, $\max .=34)$. Die kumulierte Gesamtexposition betrug im Mittel 0,217 mg/m $\mathrm{m}^{3}$-Jahre ( $\mathrm{min} .=0,005$, max. $=0,941)$. Der Mittelwert der Cyanurchloridkonzentration pro Expositionsjahr lag bei $0,020 \mathrm{mg} / \mathrm{m}^{3}$ ( $\mathrm{min} .=0,003$, $\max .=0,051)$.

Die Auswertung der Messwerte zum spezifischen IgE ergab Hinweise auf eine Sensibilisierung gegenüber Cyanurchlorid. Für die geschlossenen Anlagen von Antwerpen und Wesseling fand sich eine niedrigere Sensibilisierungsprävalenz als in der offenen Anlage von Münchsmünster. In Regressionsanalysen ist zu klären, ob dieser Unterschied statistisch zu sichern ist, die beschriebene Sensibilisierung mit einer Allergisierung der Atemwege einhergeht und sich eine Abhängigkeit von der Exposition einstellt. Für eine Bewertung ist allerdings zu berücksichtigen, dass die Gruppe der untersuchten Studienteilnehmer durch die Querschnittbeschränkung und die zusätzliche Selbstselektion (Zustimmung zur Blutabnahme) doppelt selektiert wurde.

Respiratorische Symptome und Erkrankungen wurden nur in geringem Umfang berichtet: Atemnot $(n=5$ Fälle) und die COPD-Stufen Ib $(\mathrm{n}=1)$, IIb $(\mathrm{n}=0)$, IIc $(\mathrm{n}=0)$, IIIa $(\mathrm{n}=8), \operatorname{IIIb}(\mathrm{n}=0)$, IVa $(n=1), I V b(n=0)$ wurden relativ selten oder gar nicht genannt. Lediglich bei Asthma $(n=33)$, chronischer Bronchitis $(n=14)$ und den COPD-Stufen Ia $(n=38)$ und IIa $(n=26)$ lagen ausreichende Fallzahlen vor, die weitergehenden Analysen im Querschnitt indizieren.

Insgesamt standen 2983 Untersuchungen der Lungenfunktionsparameter VC, FVC und $\mathrm{FEV}_{1}$ zur Verfügung. Im Durchschnitt nahm jeder Studienteilnehmer an 7,6 medizinischen Untersuchungen mit Lungenfunktionsmessungen teil. Die VC in Prozent vom EU-Sollwert nach Quanjer et al. [46] betrug bei der ersten (letzten) Lungenfunktionsuntersuchung jedes Studienteilnehmers im Mittel 100\% (103\%), die FVC 103\% (103\%), die $\mathrm{FEV}_{1}$ 99\% (99\%) und die $\mathrm{FEV}_{1} \% \mathrm{FVC}$ lag bei 99\% (99\%). Dieser Vergleich der gemessenen Lungenfunktionswerte mit den EU-Sollwerten nach Quanjer et al. [46] 
ergab somit für keinen der betrachteten Parameter eine Auffälligkeit. Im Mittel liegen die untersuchten Kollektive im Normalbereich. Die FVC in Prozent vom Referenzwert nach GLI betrug bei der ersten (letzten) Lungenfunktionsuntersuchung jedes Studienteilnehmers im Mittel 95,7\% (95,8\%), die $\mathrm{FEV}_{1}$ 94,4\% (93,9\%) und die $\mathrm{FEV}_{1} \% \mathrm{FVC}$ lag bei $98,2 \%$ (97,6\%). Die verschiedenen Referenzwerte (Quanjer, GLI) ergaben somit geringe Unterschiede, jedoch fand sich einheitlich kein Hinweis auf eine Abnahme der auf die Sollwerte bezogenen Lungenfunktionsparameter mit der Expositionsdauer (Vergleich der Ergebnisse zu letzter und erster Lungenfunktionsuntersuchung).

Der Anteil der Studienteilnehmer, die bei der ersten Untersuchung jünger als 25 Jahre waren, betrug 21,6\% $(n=85)$. Der Anteil der Studienteilnehmer, die bei der letzten Untersuchung jünger als 25 Jahre waren, betrug $2,3 \%(n=9)$. Aus physiologischer Sicht hatten diese Studienteilnehmer noch nicht ihr maximales Lungenvolumen erreicht. Die von Quanjer et al. [46] vorgeschlagene Vorgehensweise sieht für Personen vor, die jünger als 25 Jahre alt sind, dass bei der Sollwertberechnung das Alter auf 25 Jahre gesetzt wird, sodass z. B. die FVC in Prozent vom Sollwert (FVC\%Sollwert) bei diesen Personen in der Regel unterhalb $100 \%$ liegt. Da der Anteil der Personen unter 25 Jahren insbesondere am Ende der Untersuchung mit 2,3\% klein ist, sollte der Vergleich mit den Quanjer-Werten [46] insbesondere für diesen Querschnitt informativ sein.

Eine interne Analyse der Lungenfunktionswerte in Abhängigkeit von der Langzeitexposition gegenüber Cyanurchlorid unter Berücksichtigung von Kovariablen ist allerdings vor einer inhaltlichen Deutung dieses Befunds notwendig, um in dieser im Mittel unauffälligen Beschreibung mögliche nachteilige Kausaleffekte der Exposition aufzuklären.

Die Darstellung der Lungenfunktionsparameter über die Zeit ergab tendenziell steigende Werte. Im Folgenden werden mögliche Erklärungen für und Folgerungen aus dieser Beobachtung erörtert. Diese Beobachtung der über die Zeit ansteigenden Werte könnte durch eine Selektion der Mitarbeiter im Sinne eines Healthy-Worker-Effekts [7] bedingt sein.
Eine solche Selektion gesünderer Arbeiter führt zu einer potenziellen Verzerrung der Querschnittuntersuchung und indiziert eine Analyse im Längsschnitt [40]. Der Anstieg der Lungenfunktionsmesswerte könnte zudem durch einen Messgerätewechsel bedingt sein. Somit sollten Zusammenhangsanalysen zwischen Cyanurchloridexposition und Lungenfunktionsparametern das jeweils verwendete Messgerät berücksichtigen. Die Lungenfunktionsmessgeräte wurden hierzu zunächst in vier Gruppen (Glockenspirometer, Vitalograph, Custo Vit und Bodyplethysmographie) klassifiziert. Im Querschnitt wurden für die weiteren Analysen nur noch drei Lungenfunktionsmessgerätegruppen als Kovariablen mitgeführt, da bei der letzten medizinischen Untersuchung nur eine Person mit dem Glockenspirometer untersucht wurde. In diesen ansteigenden Lungenfunktionsmesswerten könnte sich jedoch auch indirekt eine nachteilige Wirkung der Cyanurchloridexpositionshöhe (Konzentration) spiegeln, die über der Zeit tendenziell abfällt (während die kumulierte Exposition weiter steigt). Dies indiziert neben Auswertungen zur kumulierten Exposition die Durchführung zusätzlicher Analysen, in denen die Expositionsdauer und -konzentration gegenüber Cyanurchlorid als getrennte Variablen eingehen.

Die Studie stellt den weltweit umfassendsten Datenbestand zur Ermittlung der gesundheitlichen Auswirkung einer Langzeitexposition gegenüber Cyanurchlorid am Arbeitsplatz bereit. Zur epidemiologischen Zusammenhangsanalyse wird in Teil 2 berichtet [41].

\section{Fazit}

Die Studie stellt den weltweit umfassendsten Datenbestand zur Ermittlung der gesundheitlichen Auswirkung einer Cyanurchloridlangzeitexposition am Arbeitsplatz bereit.

- Für eine Studiengruppe von 394 Männern, die zwischen 1958 und 2007 in cyanurchloridproduzierenden Betrieben tätig waren, wurden in einer retrospektiven Kohortenstudie medizinische Daten, Tätigkeiten und Expositionen erfasst und verarbeitet.
- Insgesamt standen 2983 Untersuchungen der Lungenfunktionsparameter VC, FVC und FEV 1 zur Verfügung. Diese Lungenfunktionswerte zeigten keine Auffälligkeiten verglichen mit den EU-Sollwerten.

\section{Korrespondenzadresse}

\section{PD Dr. P. Morfeld}

Institut für Epidemiologie und Risikobewertung in der Arbeitswelt, Evonik Industries AG Rellinghauser Str. 1-11, 45128 Essen peter.morfeld@evonik.com

Danksagung. Wir bedanken uns bei den Mitarbeitern, die an der Untersuchung teilnahmen und bei der Business Unit Industrial Chemicals (BU IC) der Evonik Industries AG für die finanzielle Unterstützung des Projekts. Das Forschungsvorhaben wurde von einem Begleitkreis betreut, der wesentlichen fachlichen Input bereitstellte, und in dem u. a. die Betriebsleitungen, der zuständige Geschäftsbereich sowie die verantwortlichen arbeitsmedizinischen Dienste vertreten waren. Insbesondere sei auch der Mitbestimmung (Herr Strobl) und dem Vertreter des Konzerndatenschutzbeauftragten (Herr Seeger) gedankt. Nur durch deren Einsatz wurde das Projekt auf Basis eines modernen Erfassungssystems realisierbar. Wir bedanken uns bei Frau Prof. Dr. Leng, Currenta GmbH \& Co. OH (ehemals: Bayer Industries Services $\mathrm{GmbH} \& \mathrm{Co} . \mathrm{OH}$ ), Institut für Biomonitoring, Leverkusen, für die Analyse der Blutseren. Wir danken einem unbekannten Reviewer für die hilfreiche Begutachtung einer früheren Manuskriptversion.

\section{Einhaltung ethischer Richtlinien}

Interessenkonflikt. Sponsor Evonik Industries AG, Business Unit Advanced Intermediates. Mitarbeiter der Business Unit Advanced Intermediates: W. Blumberg, E. Gross u. D. Strobl. Mitarbeiter der Evonik Evonik Industries AG, aber nicht der Business Unit Advanced Intermediates: R. Bauden, U. Küpper, P. Morfeld u. B. Noll. Die Business Unit "Advanced Intermediates" der Evonik Industries AG produziert Cyanurchlorid (s. www.evonik.com bzw. http://corporate.evonik.com/ de/unternehmen/profil-organisation/operative-bereiche/advanced-intermediates/Pages/default.aspx).

Dieser Beitrag beinhaltet keine Studien an Menschen oder Tieren.

\section{Literatur}

1. AQura GmbH (2005) Cyanurchlorid - Messungen am Arbeitsplatz. Report, Hanau

2. Attfield MD, Schleiff PL, Lubin JH et al (2012) The diesel exhaust in miners study: a cohort mortality study with emphasis on lung cancer. J Natl Cancer Inst 104:869-883 
3. Bacaloglu R, Fliegel E, Ostrogovich G (1971) Quantitative Bestimmung von Cyanurchlorid im Gemisch mit seinen Hydrolyseprodukten und anderen Polymeren des Chlorcyans. Anal Bioanal Chem 257:273-274

4. Bartels H, Bücherl E, Hertz C et al (1959) Lungenfunktionsprüfungen. Methoden und Beispiele klinischer Anwendung. Springer, Berlin

5. Beane Freeman LE, Blair A, Lubin JH et al (in Vorbereitung) Mortality from solid tumors among workers in formaldehyde industries: an update of the $\mathrm{NCl}$ cohort. Am J Ind Med. doi:10.1002/ajim.22214 (Epub ahead of print)

6. Beratergremium für umweltrelevante Altstoffe (BUA) der Gesellschaft Deutscher Chemiker (1994) Cyanurchlorid (2,4,6-Trichlor-1,3,5-triazin) BUAStoffbericht 125 (Stand: August 1993). S. Hirzel, Stuttgart

7. Checkoway H, Pearce N, Kriebel D (2004) Research methods in occupational epidemiology, 2. Aufl. Oxford University Press, New York

8. Creely KS, Cowie H, Van Tongeren M et al (2007) Trends in inhalation exposure - a review of the data in the published scientific literature. Ann Occup Hyg 51:665-678

9. Dahmann D, Taeger D, Kappler M et al (2008) Assessment of exposure in epidemiological studies: the example of silica dust. J Expo Sci Environ Epidemiol 18:452-461

10. Dearman RJ, Smith S, Basketter DA, Kimber I (1996) Classification of chemical allergens according to cytokine secretion profiles of murine lymph node cells. J Appl Toxicol 17:53-62

11. DFG (Deutsche Forschungsgemeinschaft) (2010) MAK- und BAT-Werte-Liste. WILEY-VCH, Weinheim

12. Diller W, Lewalter J, Hoffarth $\mathrm{H}$ et al (1992) Berufsasthma durch Cyanurchlorid. In: Kreutz R, Piekarski C (Hrsg) Dokumentationsband über die 32 Jahrestagung der Deutschen Gesellschaft für Arbeitsmedizin und Umweltmedizin eV (DGAUM) in Köln, 18-28 Mai 1992. Gentner, Stuttgart, S. 102-104

13. EU (2006) Regulation (EC) No $1907 / 2006$ of the European Parliament and of the Council of 18 December 2006 concerning the Registration, Evaluation, Authorisation and Restriction of Chemicals (REACH), establishing a European Chemicals Agency, amending Directive 1999/45/EC and repealing Council Regulation (EEC) No 793/93 and Commission Regulation (EC) No 1488/94 as well as Council Directive 76/769/EEC and Commission Directives 91/155/EEC, 93/67/EEC, 93/105/EC and 2000/21/ EC

14. EU (2008) Regulation (EC) No $1272 / 2008$ of the European Parliament and of the Council of 16 December 2008 on classification, labelling and packaging of substances and mixtures, amending and repealing Directives 67/548/EEC and 1999/45/ EC, and amending Regulation (EC) No. 1907/2006

15. Fruhmann G, Woitowitz H-J (1997) Chronisch-obstruktive Bronchitis und Lungenemphysem. Dtsch Arztebl 94:192-193

16. GESTIS-Stoffdatenbank des IFA (2011) 2,4,6-Trichlor-1,3,5-triazin. http://gestis.itrust.de/nxt/gateway.dll?f=templates\&fn=default.htm\&vid=gestisdeu:sdbdeu. Zugegriffen: 15.07.2013

17. GLI (2012) Global Lung Function Initiative. http:// www.lungfunction.org. Zugegriffen: 15.07.2013

18. GOLD (2010) Spirometry for health care providers. http://www.gpcme.co.nz/pdf/GOLD\%20SpirometryFull[1].pdf. Zugegriffen: 15.07.2013
19. GOLD (2011) Global strategy for the diagnosis, management, and prevention of chronic obstructive pulmonary disease. http://www.goldcopd.org/ uploads/users/files/GOLD_Report_2011_Feb21. pdf. Zugegriffen: 15.07.2013

20. Harber P, Muranko H, Shvartsblat S et al (2003) A triangulation approach to historical exposure assessment for the carbon black industry. J Occup Environ Med 45:131-143

21. Hartwig A (2010) Gesundheitsschädliche Arbeitsstoffe. Toxikologisch-arbeitsmedizinische Begründungen von MAK-Werten und Einstufungen. In: DFG (Deutsche Forschungsgemeinschaft) (Hrsg). WILEY-VCH, Weinheim

22. Hornung RW, Reed LD (1990) Estimation of average concentration in the presence of nondetectable values. Appl Occup Environ Hyg 5:46-51

23. Ignacio JS, Bullock WH (2006) A strategy for assessing and managing occupational exposures, 3. Aufl. In: Association AlH (Hrsg). AlHA, Fairfax

24. ImmunoCAP (2006) Specific IgE. Fluoroenzymeimmunoassay. Phadia $A B$, Schweden

25. ImmunoCAP (2006) Total lgE. Fluoroenzymeimmunoassay. Phadia AB, Schweden

26. ISIC (2005) United Nations' international standard industrial classification. http://unstats.un.org/ unsd/cr/registry/regct.asp? Lg=1. Zugegriffen: 15.07.2013

27. Jedrychowski R (1994) Cyanuric chloride (2,4,6-trichloro-1,3,5-trazine 90-days repeated exopsure inhalation toxicity study in rats). The Nofer Institute of Occupational Medicine, Lodz

28. Jedrychowski RA, Gorny R, Stetkiewicz J, Stetkiewicz I (1992) Subacute oral toxicity of cyanuric chloride in the rat. Toxicologist 12:119 (Abstract)

29. Jones M, Graham C, Taylor AN et al (1998) Immunologic cross-reactivity between respiratory chemical sensitizers: reactive dyes and cyanuric chloride. J Allergy Clin Immunol 102:835-840

30. Kaskevich LM, Soboleva LP, Kolpakov IE et al (1984) Nature of the effect of cyanuric chloride on the human body. Vrach Delo 7:109-112

31. Kasper DL, Fauci AS, Longo DL et al (2005) Harrison's principles of internal medicine, 16. Aufl. McGraw-Hill, New York

32. Konietzko N, Wendel H, Wiesner B (1995) Erkrankungen der Lunge. Walter de Gruyter \& Co., Berlin

33. Kramer T, Periclès-Bürgisser (1992) Bestimmung von Cyanurchlorid in der Luft. Report Ciba-Geigy. Zentrales Arbeitshygienelabor, Basel

34. Kriebel D, Checkoway H, Pearce N (2007) Exposure and dose modelling in occupational epidemiology. Occup Environ Med 64:492-498

35. Kunz K, Groß E (2009) Determination of the cyanuric chloride concentration in a full face gas mask. Contract No.: 2009-7123

36. Loomis D, Kromhout $\mathrm{H}$ (2004) Exposure variability: concepts and applications in occupational epidemiology. Am J Ind Med 45:113-122

37. Mannebeck D, Schmidt M, Munder A, Vormberg $R$ (1999) Bestimmung des Geruchsschwellenwertes für Cyanurchlorid. GIT Labor-Fachzeitschrift 43:121-124 (Sonderdruck)

38. McFadden ER (2004) Asthma. In: Kasper DL, Fauci AS, Longo DL et al (Hrsg) Harrison's principles of internal medicine, 16. Aufl. McGraw-Hill, New York, S. $1508-1516$

39. Mertschenk B, Burkhart-Reichl A, Ergenzinger $M$ et al (1998) Cyanurchlorid - arbeitsmedizinisch-toxikologische Bewertung der Exposition in der Produktion unter Aspekten der Arbeitssicherheit. Zentralbl Arbeitsmed 48:504-510
40. Morfeld P (2005) Zur Analyse longitudinaler epidemiologischer Daten aus der Arbeitswelt. In: Bundesanstalt für Arbeitsschutz und Arbeitsmedizin (Hrsg) Arbeitsbedingte Erkrankungen der Atemwege Tagungsbericht 143. Wirtschaftsverlag NW, Dortmund, S. 20-37

41. Morfeld P, Noll B (2014) Cyanurchloridbelastung in Produktionsbetrieben - Teil 2: Querschnittsanalysen zu respiratorischen Symptomen und Längschnittanalyse zu Lungenfunktionsparametern. Zbl Arbeitsmed Arbeitsschutz Ergon DOI 10.1007/ s40664-013-0002-4 (in Druck)

42. Munder A, Jung H (1993) Ringversuch zur Cyanurchlorid-Bestimmung. Contract No.: U 263/93

43. Munder A, Krügel R, Günther K (1999) Gehaltsbestimmung von Cyanurchlorid in der Luft nach adsorptiver Probenahme und Hydrolyse zur Cyanursäure mittels HPLC

44. OECD SIDS (2001) SIDS dossier on the HPV chemical cyanuric chloride. CAS No. 108-77-0. Bern, Switzerland

45. Pleger E, Müller E, FrankW, Bender J (1991) Die Oszillationsmethode - eine kostengünstigere Alternative für die atemmechanische Funktionsdiagnostik in der Praxis? Z Arztl Fortbild 85:187-192

46. Quanjer PH, Tammeling GJ, Cotes JE et al (1993) Lung volumes and forced ventilatory flows. Report working party standardization of lung function tests, European community for steel and coal. Official statement of the European respiratory society. Eur Respir J Suppl 16:5-40

47. Sabbioni G, Wesp H, Lewalter J, Rumler R (2007) Determination of isocyanate biomarkers in construction site workers. Biomarkers 12:468-483

48. Seixas NS, Checkoway H (1995) Exposure assessment in industry specific retrospective occupational epidemiology studies. Occup Environ Med 52:625-633

49. Silverman DT, Samanic CM, Lubin JH et al (2012) The diesel exhaust in miners study: a nested casecontrol study of lung cancer and diesel exhaust. J Natl Cancer Inst 104:855-868

50. Ulmer WT (1998) Lungenfunktions-Manual. Thieme, Stuttgart

51. Ulmer WT, Reichel G, Nolte D, Islam MS (1991) Die Lungenfunktion. Physiologie und Pathophysiologie, Methodik, 5. überarbeitete Aufl. Thieme, Stuttgart

52. Vance JW (1963) A bellows-type spirometer for measuring the forced expirogram. Chest 43:57-61

53. Vitalograph (1973) Lungenventilationsmessung ein einfaches Verfahren. Ein Handbuch zur Untersuchung der Ventialtionsfähigkeit mit dem Vitalograph-Keilbalgspirometer. Handbuch. Vitalograph Ltd, Buckingham

54. WHO (1961) Report of an expert committee: definition and diagnosis of pulmonary disease with special reference to chronic bronchitis and emphysema. Technical report series.

55. Yawn BP (2008) Factors accounting for asthma variability: achieving optimal symptom control for individual patients. Prim Care Respir J 17:138-147 